\title{
Full Parametric Impedance Analysis of Photoelectrochemical Cells: Case of a $\mathrm{TiO}_{2}$ Photoanode
}

\author{
Hung Tai Nguyen*, Thi Lan Tran*, Dang Thanh Nguyen*,t, \\ Eui-Chol Shin**, Soon-Hyung Kang**, and Jong-Sook Lee*, \\ ${ }^{*}$ School of Materials Science and Engineering, Chonnam National University, Gwangju 61186, Korea \\ **Department of Chemistry Education, Chonnam National University, Gwangju 61186, Korea
}

(Received January 10, 2018; Revised May 9, 2018; Accepted May 9, 2018)

\begin{abstract}
Issues in the electrical characterization of semiconducting photoanodes in a photoelectrochemical (PEC) cell, such as the cell geometry dependence, scan rate dependence in DC measurements, and the frequency dependence in AC measurements, are addressed, using the example of a $\mathrm{TiO}_{2}$ photoanode. Contrary to conventional constant phase element (CPE) modeling, the capacitive behavior associated with Mott-Schottky (MS) response was successfully modeled by a Havriliak-Negami (HN) capacitance function-which allowed the determination of frequency-independent Schottky capacitance parameters to be explained by a trapping mechanism. Additional polarization can be successfully described by the parallel connection of a Bisquert transmission line (TL) model for the diffusion-recombination process in the nanostructured $\mathrm{TiO}_{2}$ electrode. Instead of shunt $\mathrm{CPEs}$ generally employed for the non-ideal TL feature, TL models with ideal shunt capacitors can describe the experimental data in the presence of an infinite-length Warburg element as internal interfacial impedance - a characteristic suggested to be a generic feature of many electrochemical cells. Fully parametrized impedance spectra finally allow in-depth physicochemical interpretations.
\end{abstract}

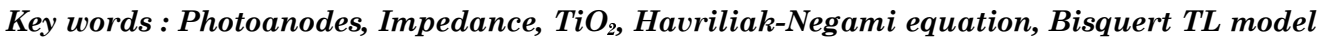

\section{Introduction}

$\mathrm{P}$ hotoelectrochemical (PEC) cells have been investigated intensively in recent years for their use as light-to-electrical energy conversion devices. Recently, Si-doped $\mathrm{TiO}_{2}$ nanorods-prepared by the hydrothermal method-have been investigated as photoanodes for water splitting. Nominally $2 \%$ Si-doped $\mathrm{TiO}_{2}$ nanorods were shown to exhibit higher performance when compared with the pristine $\mathrm{TiO}_{2}{ }^{1}{ }^{1}$ In this work, a similarly prepared $5 \%$ Si-doped $\mathrm{TiO}_{2}$ sample was examined in a different experimental setup. Electrical characterizations using nonstandardized, three-electrode, photoelectrochemical setups are plagued with artefacts depending on the chamber geometry, position of electrodes, illumination conditions, etc., and thus are poorly reproducible and often incompatible. $\left.{ }^{2}\right)$ Using this work as an example, several general issues regarding the electrical characterization of PEC cells are addressed.

Typical electrical characterizations of PEC cells include DC and AC analyses: 1) (Direct) current-voltage (or potential) $(I-V)$ characteristics measured often with a chopped

\footnotetext{
Presently at Samsung Display, Vietnam

Presently at Samsung SDI, Korea

${ }^{\S}$ Corresponding author: Jong-Sook Lee

E-mail : jongsook@jnu.ac.kr

Tel : +82-62-530-1701 Fax : +82-62-530-1699

The authors certify that there is no authorship dispute between them.
}

white light source for PEC performance over a potential range of interest. 2) Incident-photon-to-current-conversionefficiency (IPCE) where photo- and dark current are similarly measured at a fixed electrochemical potential over the spectral range of interest. The efficiency is estimated with respect to the response of the reference silicon photodiode. 3) Capacitance-voltage $(C-V)$ characteristics for MottSchottky (MS) analysis conventionally measured at chosen fixed frequencies of AC oscillations, usually in dark conditions, similarly as for the semiconductor junctions in solid state electronic devices. ${ }^{3)}$ As the semiconductor/liquid electrolyte junctions are easily made, MS analysis may be conveniently applied to a PEC configuration for semiconductor characterizations.

$\mathrm{TiO}_{2}$ electrodes are also the photoelectrodes of dye-sensitized solar cells (DSSCs). $\mathrm{TiO}_{2}$ electrodes in contact with organic electrolytes for DSSCs may suggest some similarity with those in PEC cells. In-depth parametric impedance spectroscopy for DSSCs with nanostructured $\mathrm{TiO}_{2}$ electrodes, developed over more than a decade, ${ }^{4-10)}$ features transmission line (TL) models for the electron diffusionrecombination process. The diffusion-recombination mechanism is suggested as being applicable for $\mathrm{TiO}_{2}$ electrodes of DSSCs which are i) low-doped, i.e. of small carrier concentration, and ii), nanostructured, so that the electron charge in the nanostructured $\mathrm{TiO}_{2}$ is compensated by the positive ion charge of the surrounding organic electrolyte and thus no electrical potential gradient or electron drift occurs. 
TL model responses are also indicated in nanostructured $\mathrm{TiO}_{2}$ in aqueous cells, as for PEC water splitting, ${ }^{9)}$ so the applicability of the TL impedance model may be considered for PEC electrodes, as in this work. As introduced above, MS analysis is a typical AC characterization of PEC cells, but usually one fixed frequency response is monitored as a function of potentials. MS analysis assumes highly doped semiconductors, where the charge depletion or accumulation is limited at the interface, regardless of the feature size. It should be noted however, that the doping concentration of the semiconductor electrodes for DSSCs or PEC cells, including this work, is seldom controlled or well-defined. Often developed in diverse meso- and nanoporous structures aiming at high performance, ${ }^{11-13)}$ photoanodes are likely to have strong microscopic inhomogeneity in the doping concentration, in surface potentials, and also in the feature size. Therefore, the diffusion-recombination mechanism in the response of a PEC electrode may not be a priori excluded. The porous nanostructured PEC electrodes may be low-doped, so that the applied potential changes the Fermi level homogeneously in the electrode layer. ${ }^{4-10)}$

In fact, the two different impedance mechanisms or models concern different relevant potential (bias) ranges: 1) MS behavior applies to the depletion of majority charge carriers in $\mathrm{TiO}_{2}$ electrodes under reverse bias; 2) The diffusionrecombination mechanism in DSSCs is most prominent under forward bias near open circuit voltage (OCV), where the charge carrier concentration in $\mathrm{TiO}_{2}$ electrodes increases.
The two mechanisms are considered together in semiconductor devices containing $p n$ junctions where the majority charge carrier depletion at the interface and minority charge carrier diffusion and storage occur. ${ }^{14,15)}$ Two capacitance terms, depletion and diffusion, are added together. The electrical behavior of organic bulk heterojunction solar cells is also described by combination of the depletion capacitance and diffusion. ${ }^{16)}$ It should be mentioned that TL model response can also be ascribed to the distributed resistor and capacitor components of porous metallic electrodes ${ }^{17)}$ or the percolating network in the insulating matrix, ${ }^{18)}$ not related to the diffusion-related processes.

In this work, therefore, the co-presence of the two mechanisms is suggested in the $\mathrm{AC}$ response of $\mathrm{a} \mathrm{TiO}_{2}$ photoanode. Importantly, it is shown how non-trivial huddles in the parametric description of two mechanisms are successfully overcome. The prospect is considered bright for the systematic examination and comprehensive understanding of the semiconducting oxides for water splitting.

\section{Experimental Procedures}

The PEC properties of $5 \%$ Si-doped, $\mathrm{TiO}_{2}$ nanorods were examined in a lab-made PEC test station reported earlier. ${ }^{13)}$ The experimental setup included a miniature PEC cell, a xenon lamp (LS-150, ABET Technologies Inc., USA), a custom-made automatic chopper, a monochromator (Spectro, Korea), and a potentio/galvanostat with a frequency

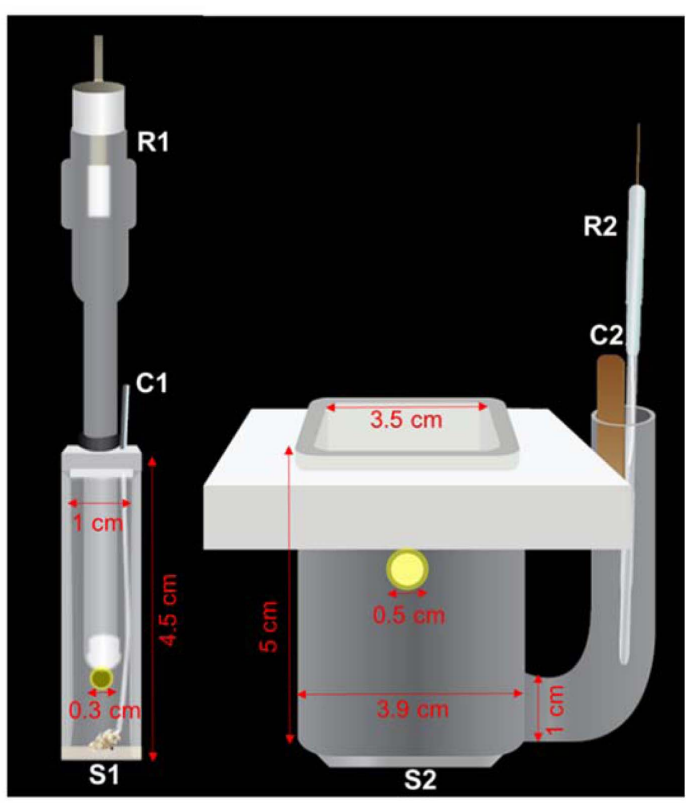

(b)

(a)

Fig. 1. (a) PEC setup with the lab-made miniature quartz cell based on an optical cuvette. The $\mathrm{TiO}_{2}$ nanorods electrode reported earlier $^{1)}$ is schematically illustrated. (b) Close-to-scale diagrams of the present setup (S1) and that from the previous report $^{1)}(\mathrm{S} 2)$. 
response analyzer (Autolab PGSTAT 302N FRA2, The Netherlands), as shown in Fig. 1(a). A close-to-scale schematic diagram of the setup is shown as $\mathrm{S} 1$ in Fig. 1(b). The miniature quartz cell was designed by drilling a hole in a commercial optical cuvette $\left(12.5 \times 12.5 \times 45 \mathrm{~mm}^{3}\right)$ where the semiconductor electrode was fixed using a rubber O-ring from outside. The $3 \mathrm{~mm}$ diameter hole size defines the electrode area in contact with the electrolyte as $7 \mathrm{~mm}^{2}$. A commercial $\mathrm{Ag} / \mathrm{AgCl}$ reference electrode (RE-ICP, ALS Co., Japan) and a Pt mesh counter electrode were used for a three-electrode configuration. Aqueous solution $0.1 \mathrm{M}$ $\mathrm{NaOH}(\mathrm{pH}=13)$ was used as an electrolyte. $I$ - $V$ characteristics were measured during the light-off and light-on cycles with the chopping period of $10 \mathrm{~s}$. Different scan rates were tested at $0.02 \mathrm{~V} \cdot \mathrm{s}^{-1}$ and $0.005 \mathrm{~V} \cdot \mathrm{s}^{-1}$. Electrochemical impedance spectroscopy (EIS) was performed as a function of the anodic potential at $0.1 \mathrm{~V}$ intervals with $\mathrm{AC}$ peak amplitude of $10 \mathrm{mV}$. The IPCE measurement was carried out for the wavelength range of $300 \mathrm{~nm}$ to $600 \mathrm{~nm}$, at a scan rate 1 $\mathrm{nm} \cdot \mathrm{s}^{-1}$. Light intensity was calibrated using a silicon reference cell (BS-500 S/N 017, Bunkoukeiki Co., Ltd, Japan).

\section{Results and Discussion}

\subsection{I-V characteristics and IPCE}

Figure 2(a) shows $I-V$ curves with scan rates $0.02 \mathrm{~V} \cdot \mathrm{s}^{-1}$, except one at $0.005 \mathrm{~V} \cdot \mathrm{s}^{-1}$ which is presented alone in Fig. 2(b) and (c), at the same light chopping period of $10 \mathrm{~s}$. Several measurements using setups $\mathrm{S} 1$ and $\mathrm{S} 2$, as shown in Fig. 1(b), were performed for the same sample. Although the results are not completely reproducible, a systematic difference between the two reference electrodes was confirmed. The increase in cathodic currents was higher with the commercial reference electrode $\mathrm{R} 1$, than $\mathrm{R} 2$, a lab-made one, which is nominally of the same structure, i.e. $\mathrm{Ag} / \mathrm{AgCl}$ in saturated $\mathrm{KCl}$. When tested together in different electrolytes, there was negligible difference in the OCVs between the two reference electrodes. The origin of the difference between the two reference electrodes under cathodic condition is not clear yet. It should be noted that the measurements with R2 suggest larger onset potentials (more negative) than those with $\mathrm{R} 1$. In fact, as shown in the inset of Fig. 2(a), all the measurements exhibit OCV at around $0.2 \mathrm{~V}$ vs. $\mathrm{Ag} / \mathrm{AgCl}$ in dark conditions, regardless of the setup and reference electrodes. The coincidence of the light-insensitive cathodic curves for the comparative measurements in different setups $\mathrm{S} 1$ and $\mathrm{S} 2$ suggests that the electrode area is well-defined by the hole sizes as $3 \mathrm{~mm}$ and $5 \mathrm{~mm}$, respectively, for $\mathrm{S} 1$ and $\mathrm{S} 2$. Other differences between the two setups should be ascribed to the quartz cell size and the position and geometry of the counter electrodes, with respect to the photoanodes, as shown in Fig. 1(b). The electrolyte volume and path length in S2 are much larger than in $\mathrm{S} 1$. An increase in dark anodic current above $1 \mathrm{~V}$ is also notable, which may be described as breakdown or leakage. Very low breakdown voltage is not uncommon in nanostructured or thin film diodes for solar cells or LEDs, and can be attributed to leakage. For the thick, single, crystalline $\mathrm{TiO}_{2}$ sample reported by Honda and Fujishima, ${ }^{19)}$ and $\mathrm{ZnO}$ single crystalline photoanodes with diffusion-bonded Pt back con-
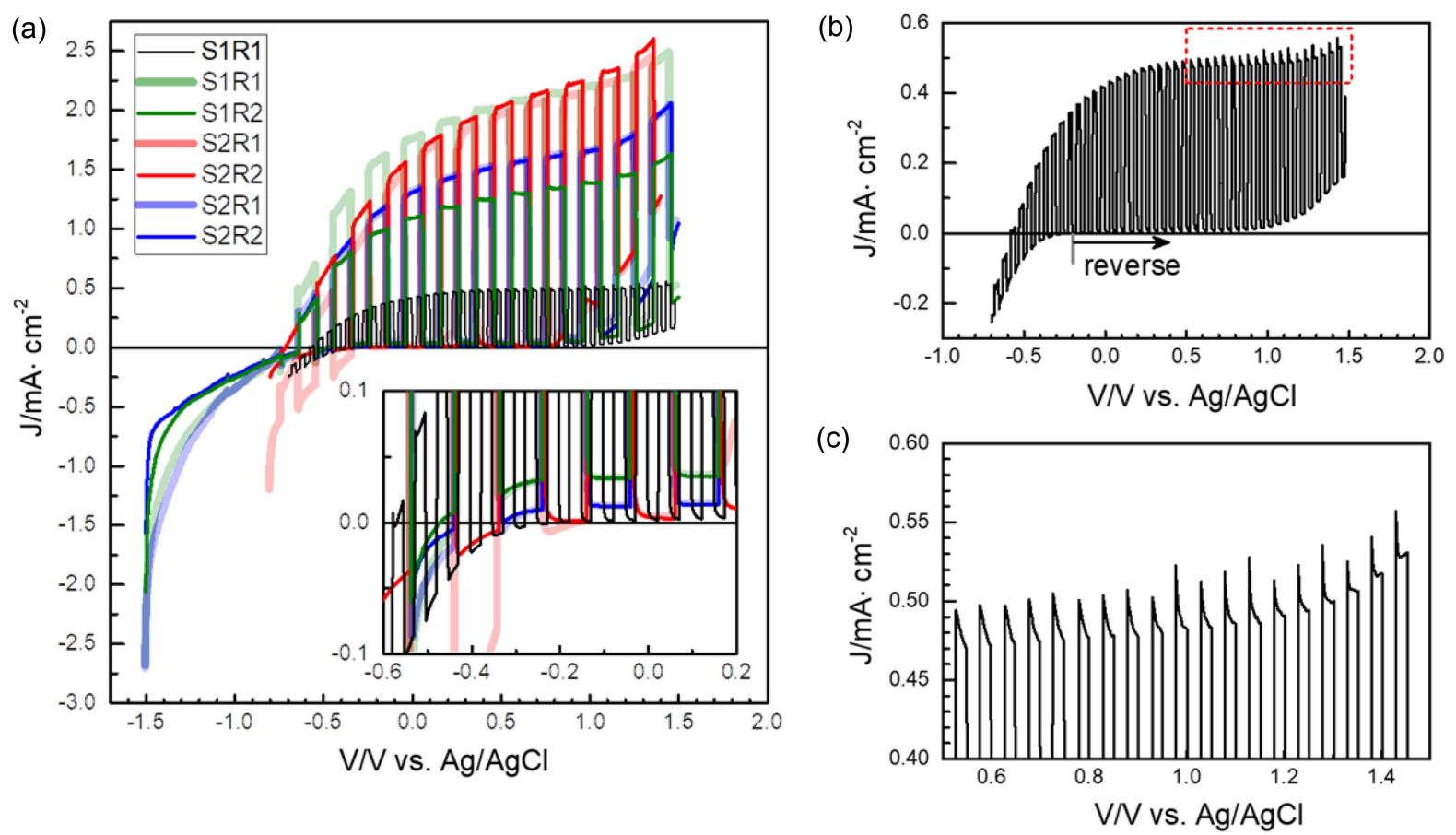

Fig. 2. (a) $I-V$ characteristics with light on/off for $10 \mathrm{~s}$ periods using different setups and different reference electrodes, as indicated in Fig. 1(b). Inset shows the magnification near OCV in the dark. The scan rates are $0.02 \mathrm{~V} \cdot \mathrm{s}^{-1}$ except as presented in (b) and (c). (b) $I-V$ curve in S1 using $\mathrm{R} 1$ at the scan rate of $0.005 \mathrm{~V} \cdot \mathrm{s}^{-1}$. (c) The enlarged image of the boxed region of Fig. 2(b). 
tact, ${ }^{20)}$ very stable reverse behavior up to a few volts were observed. On the other hand, very leaky $I-V$ behavior was observed for the $\mathrm{ZnO}$ films prepared by the solution method, which was moderated by the formation of a denser seed layer. ${ }^{13)}$ The larger leakage current density for the larger electrode area of S2 appear to support the nature of the breakdown spots, in contrast to the behavior of cathodic currents proportional to the electrode area. The difference in the anodic photocurrents between setups shows unequal illumination conditions. The very small electrolyte volume in the $\mathrm{S} 1$ setup appears to cause greater irreproducibility in the dynamic measurements.

$I-V$ characteristics also depend on the scan rates and the light chopping speed or periods. Slower scan rates generally reduce the photocurrents, as well as dark currents, as shown by the data scanned at $0.005 \mathrm{~V} \cdot \mathrm{s}^{-1}$ in Fig. 2(b). The spikes of the photocurrents for the boxed region are shown more clearly in Fig. 2(c). They were attributed to the accumulated photoexcited holes at the semiconductor-electrolyte interface. $^{21,22)}$ The spiky behavior is pronounced at the high reverse bias above $0.7 \mathrm{~V}$ (vs. $\mathrm{Ag} / \mathrm{AgCl}$ ). With the constant voltage scan rate of $0.005 \mathrm{~V} \cdot \mathrm{s}^{-1}$, the relaxations are shown to be faster at high reverse bias, which seems contradictory to the discussion attributing them to the accumulated photoexcited holes at the semiconductor-electrolytes interface. The increasing contribution of the fast relaxation may be related to the increasing high frequency ohmic contribution, $R_{O H M}$, distinguished by the impedance spectroscopy presented in the next section. Strong scan rate dependence can be ascribed to slow relaxation of the electrochemical response in PEC cells, corresponding to the low frequency impedance behavior. There is also often temporal change involved, since non-zero DC currents cause the evolution in mass and charge concentration in bulk, and at interfaces, and the irreversible or very slowly recovering drift in the cell responses occurs. The situation is in contrast to photovoltaic solar cells associated with much faster electronic relaxations only.

Figure 3 presents IPCE spectra measured at different potentials. The IPCE exhibits the onset wavelength at approximate $454 \mathrm{~nm}$, enabling an energetic bandgap of 2.74 $\mathrm{eV}$ to be calculated. The maximum IPCE of the sample is about $26 \%$. The IPCE is shown slightly higher at $0.5 \mathrm{~V}$ than at $0.3 \mathrm{~V}$, and both are distinctly higher than that at $0 \mathrm{~V}$. It should be noted that the potential dependence simply reflects the photocurrents in Fig. 2, since IPCE is nothing other than the net photocurrents normalized with respect to the response of the silicon standard cell with the calibrated light intensities. In this work, almost continuous scans at 1 $\mathrm{nm} \cdot \mathrm{s}^{-1}$ and at $1 \mathrm{~nm}$ steps were made, so that the spectra provided the detailed wavelength dependence. The continuously measured spectra were found to be comparable in their peak shapes and magnitudes with previously report$\mathrm{ed}^{1)}$ stepwise measurements. In steady-state measurements, values are taken at each wavelength after some relaxation time, e.g., at $20 \mathrm{~nm}$ intervals. ${ }^{1)}$ The photocur-

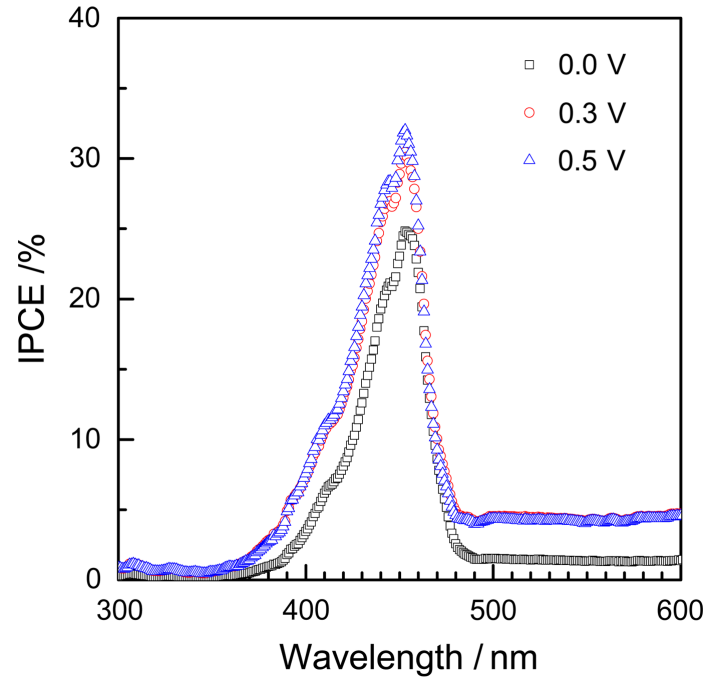

Fig. 3. IPCE spectra measured at different potentials of 0.0 $\mathrm{V}, 0.3 \mathrm{~V}$ and $0.5 \mathrm{~V}$ vs. $\mathrm{Ag} / \mathrm{AgCl}$ reference electrode at constant scan rate of $1 \mathrm{~nm} \cdot \mathrm{s}^{-1}$.

rents under monochromated illumination for IPCE exhibit relaxations or time-dependence, similarly as those under full sun condition discussed above. Steady-state currents should be taken at each potential after some transient behaviors. The waiting time is often fixed, e.g. $5 \mathrm{~s}$, for the systematic automated measurement. IPCE measurements of PEC cells therefore have the same inherent issues discussed above for the $I$ - $V$ characteristics. IPCE spectra measured continuously, as in Fig. 3, may be recommended over the broken spectra from step-wise measurements. With the experimental scan rates given and taken into consideration, the detailed wavelength dependence, such as peaks and shoulders, provides rich information on the electronic structure of photoanodes.

\subsection{Mott-Schottky Analysis and Issues}

Mott-Schottky (MS) analysis is based on the response of the Schottky space charge depletion region of the semiconducting photoanode, which behaves as a capacitor with the thickness of the depletion layer at the interface with the electrolyte controllable by external potentials. The capacitance-voltage $(C-V)$ characteristics obtained by a frequency response analyzer (FRA) combined in a potentio/galvanostat, well-known for the similarly generated depletion layer at semiconductor/metal contact, follow the relationship shown in Eq. (1):

$$
C_{S B}^{-2}=\frac{2}{q A^{2} \varepsilon_{R} \varepsilon_{0} N_{D}}\left(V_{F B}+V-\frac{k T}{q}\right)
$$

The analysis provides the donor concentration $N_{D}$ of the semiconducting anode from the slope, and the flatband potential $V_{F B}$ from the intercept. Although MS analysis is routinely performed for the characterization of PEC cells, there are problematic issues. In contrast to the flat semiconductor/metal Schottky junction, porous nanostructured pho- 


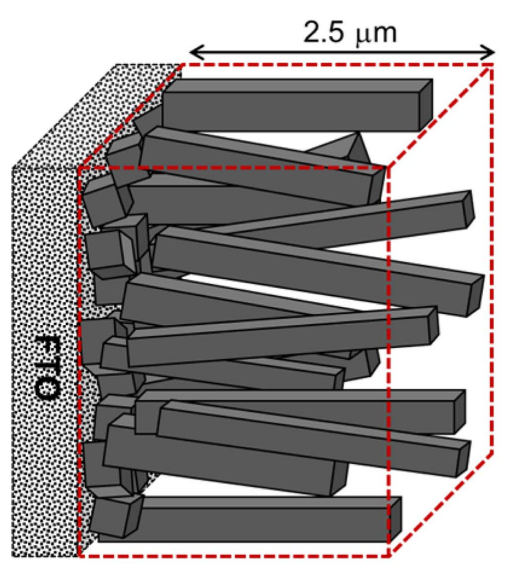

(a)
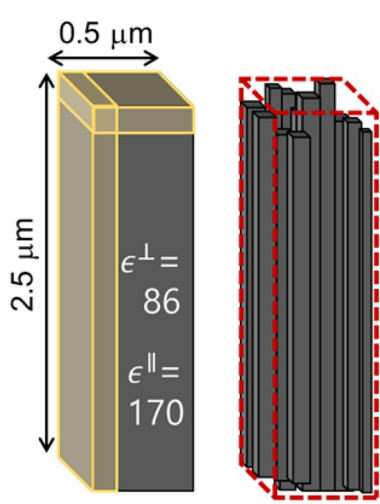

(b)

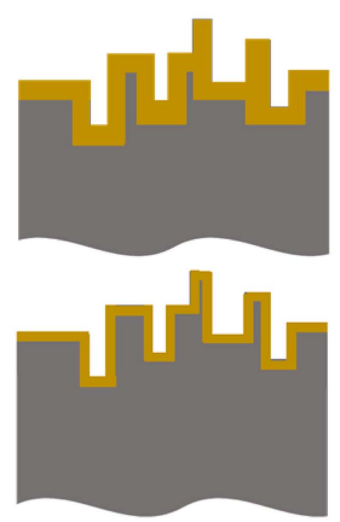

(c)

Fig. 4. (a) Schematic diagram of $\mathrm{TiO}_{2}$ nanorods grown on FTO glass. ${ }^{1)}$ (b) The crystallographic and geometrical feature of $\mathrm{TiO}_{2}$ nanorods. (c) Different thicknesses of the depletion space charge layers on a rough surface.

toanodes in PEC cells do not provide well-defined area $A$ in Eq. (1). The high performance of the nanostructured electrodes, e.g. photocurrent density $J$ in Fig. 2, is often firstly ascribed to the enlarged effective area, much larger than the macroscopic electrode area evaluated e.g. from the holes with diameter $3 \mathrm{~mm}$ or $5 \mathrm{~mm}$ in Fig. 1(b). The area $A$ is directly involved in the estimation of $N_{D}$ for the given capacitance response, thus $N_{D}$ estimates can be several orders different, depending on the estimation of $A$. The exact evaluation of the interface area is generally difficult. The situation is illustrated for the present system in Fig. 4. For the typical $\mathrm{TiO}_{2}$ nanorods of $0.5 \times 0.5 \times 2.5 \mu \mathrm{m},{ }^{1)}$ the top surface area of $0.25 \mu \mathrm{m}^{2}$ is only $5 \%$ of the lateral side area of 5 $\mu \mathrm{m}^{2}$.

For a $\mathrm{TiO}_{2}$ system, the dielectric constant $\varepsilon_{R}$ in Eq. (1) also needs some consideration. For most covalent or ionic semiconductors, the static dielectric constants $\varepsilon_{R}$ around 10 are contributed by the atomic (electronic) and ionic contribution, which are independent of frequencies in the range concerned here, and also of temperature. $\mathrm{TiO}_{2}$ in this work, however, similarly as $\mathrm{SrTiO}_{3}$ and $\mathrm{KTaO}_{3}$, is classified as incipient ferroelectric material, based on the experimental observation that its dielectric permittivity $\varepsilon_{R}$ is exceptionally large, and increases with decreasing temperature. ${ }^{23)}$ At $300 \mathrm{~K}, \varepsilon_{R}$ values along the $c$-direction and $a$-direction of the tetragonal rutile structure are 170 and 86 respectively. On the other hand, strong pseudo-dielectric effects due to electronic and ionic conduction mechanisms are also observed in the $\mathrm{TiO}_{2}$ system, which increases with increasing temperature. ${ }^{24)}$ As emphasized recently for ionic conductors, ${ }^{25-28)}$ these apparent dielectric effects from mobile charge carriers should be distinguished from the true dipolar polarization considered for the dielectric constant $\varepsilon_{R}$ in Eq. (1). Therefore, it may not always be clear which dielectric constants should be used or how to consider possible anisotropic effects. Although the evaluation of $V_{F B}$ is not formally affected by $A$ or $\varepsilon_{R}$ in Eq. (1), $V_{F B}$ can also vary microscopi- cally due to the different surface structure and chemistry of porous electrodes.

There are also thickness effects. For thin film electrodes, saturation of the depletion capacitance occurs under high reverse bias. Similarly, as illustrated in Fig. 4(c), the effective surface area of the nanostructured electrodes decreases with increasing depletion thickness, upon high reverse bias. This was suggested to explain the upwardly curved MS plot of nanostructured $\mathrm{Fe}_{2} \mathrm{O}_{3}$ electrodes, ${ }^{29)}$ and the roughness at the top surface of $\mathrm{TiO}_{2}$ nanorods, ${ }^{1)}$ as schematically shown in Fig. 4(b), may give rise to similar outcomes.

All these issues, related to poorly defined geometry factors of the nanostructured photoanodes, are very specific and difficult to be address precisely. Microstructural features that are complicatedly heterogeneous may explain the copresence of the space charge depletion effects for the highly doped semiconductors and the diffusion-recombination kinetics described by the TL model and expected for the lowdoped nanostructured electrodes, are discussed in later sections (Sec. $3.4 \& 3.5$ ).

\subsection{Capacitance Spectra and the Havriliak-Negami Equation}

A more general critical issue in MS analysis is the socalled frequency dispersion, or the frequency dependence, of the measured capacitance. The real capacitance values are directly given by the relationship with impedance data as $C^{*}=\left(j \omega Z^{*}\right)^{-1}=C^{\prime}-j C^{\prime \prime}$ for a measured frequency $f=\omega / 2 \pi$. If the system behaves as an ideal capacitor of capacitance $C$ with impedance $(j \omega C)^{-1}$, the same capacitance results, regardless of the measured frequency values chosen. However, frequency-dependent capacitances and nonlinear MS relationships have been observed even in well-defined single crystalline electrodes, ${ }^{30,31)}$ let alone in various nanostructured electrodes. Therefore, the capacitance component evaluated at an arbitrary frequency, e.g. $1 \mathrm{kHz}$, could not properly represent the Schottky capacitance $C_{S B}$ in Eq. (1) 

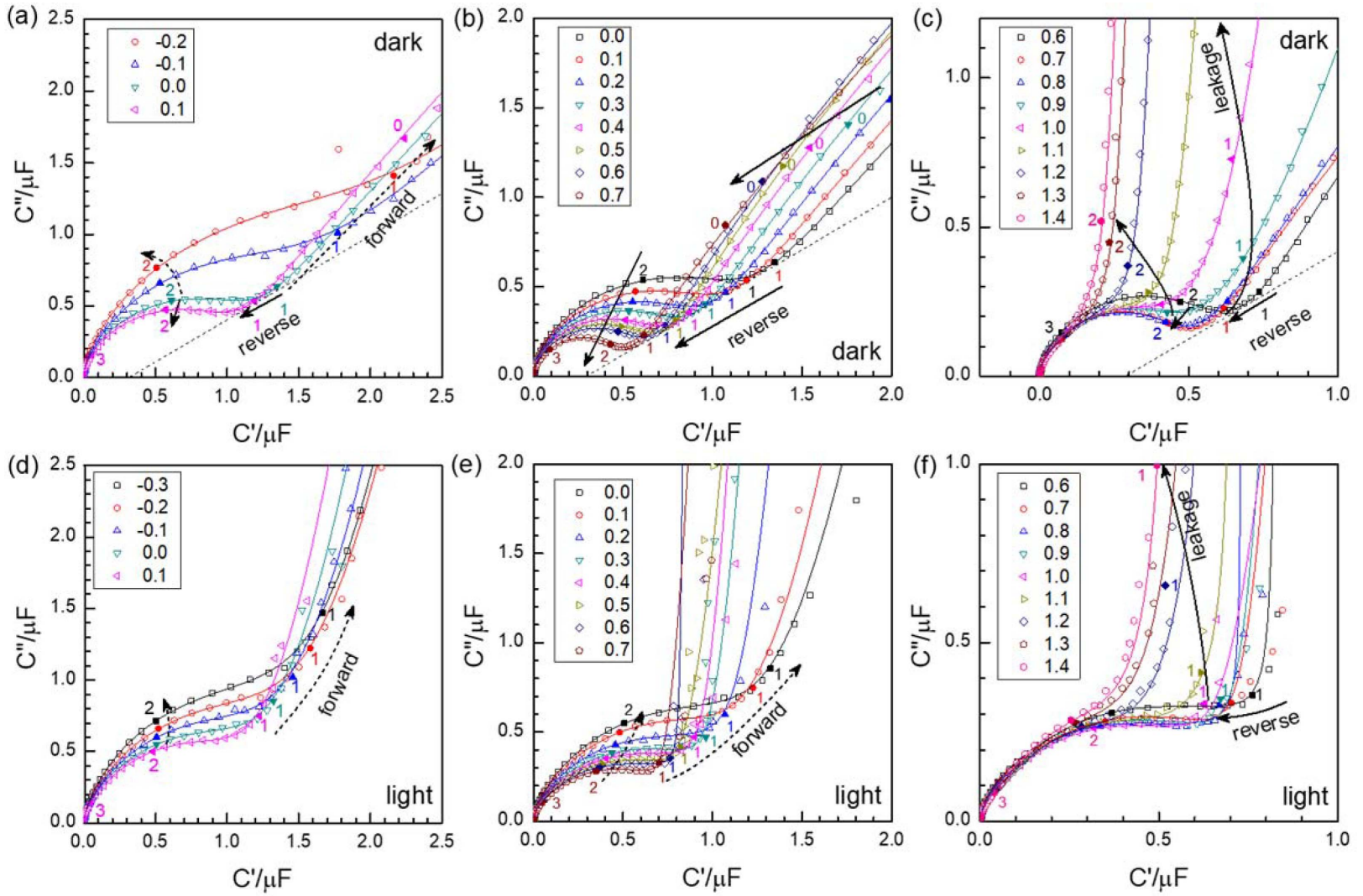

Fig. 5. Complex capacitance spectra in dark (a, b, c) and illuminated 'light' conditions (d, e, f); Near flat band voltage (a, d); reverse bias (b, e) and high reverse bias or leakage region (c, f). The numbers on the close symbols represent the logarithmic frequency values. The lines are simulated spectra using the fit results shown in Fig. 6.

for the depletion layer at the semiconductor/electrolyte interface.

Parametrized capacitance obtained by impedance model analysis could address the frequency dispersion issue. A few parametric impedance spectroscopy results for $C_{S B}$ components have been reported. ${ }^{29,32,33)}$ There is a common criticism, however, that equivalent circuit parameters are strongly model-dependent. Currently, in many different subjects and systems, including PEC cells, the impedance model analysis employs so-called CPEs (constant-phase-elements) of the complex capacitance $C_{Q}^{*}=Q(j \omega)^{\alpha-1}$, or impedance $Z_{Q}^{*}=\left(Q(j \omega)^{\alpha}\right)^{-1}$. With $\alpha=1,0, \frac{1}{2}$ the element corresponds to an ideal capacitor, a resistor, and an $R C$ TL model of infinite length, respectively. At the present, CPEs are mostly employed as 'generalized' capacitors to address the deviation from the response described by $R$ and $C$ components with $\alpha$ close to 1, e.g. 0.9. There is no circuit analog for such general $\alpha$ values. For convenience, CPE is sometimes denoted as ' $Q$ ' element. The resistance effects are then well defined in terms of the ideal $R$ elements in the model, but capacitance values of present interest are not straightforwardly provided. The widely adopted practice for estimation of effective capacitance ' $C$ ' is to use the peak frequency or time constant $\tau=(R Q)^{1 / \alpha}$ for the $(R Q)$ parallel circuits, as $\tau=R^{\prime} C$ '. This may represent a kind of mean value for the distributed relaxations, ${ }^{34)}$ but little further theoretical significance can be provided.

Since the Schottky junction is largely capacitive and highly resistive (see Fig. 9 below), the impedance plane presentation of the AC response of PEC cells under anodic condition is not very informative. The capacitance information is better shown by the complex capacitance plane graphs presented in Fig. 5. In this work, the impedance is measured from $1 \mathrm{MHz}$ to $0.1 \mathrm{~Hz}$ in logarithmic sweeps at the voltage interval of $0.1 \mathrm{~V}$. While the resistor-capacitor parallel connection, or $(R C)$, leads to the semicircular arc in the impedance plane, the capacitance arcs distinguished in all the spectra may be roughly described by the resistor-capacitor series circuit or 'Debye' circuit which is overlapped by the lower frequency response. (The resistor component is the high frequency ohmic response best distinguished in the high frequency region of the impedance spectra, as indicated in Figs. 9(d) and (h)). Systematic variation according to the MS relation is best shown in Fig. 5(b), for the potential range between $0.0 \mathrm{~V}$ and $0.6 \mathrm{~V}$, where the capacitance decreases with increasing reverse bias. It is also notable that the local minima near $10 \mathrm{~Hz}($ or $\log (10 / \mathrm{Hz})=1)$, bounding the high frequency capacitance arc from the lower frequency response, lie on one line represented by the dashed line. Systematic variation can be found at different 
frequencies, e.g. at $100 \mathrm{~Hz}($ or $\log (100 / \mathrm{Hz})=2)$. MS plots for the frequencies 10, 100, and $251 \mathrm{~Hz}$ are shown in Fig. 8(c). MS linear relationships may be found for the plots at 10 and $100 \mathrm{~Hz}$, but with different slopes and intercepts thus resulting in different $N_{D}$ and $V_{F B}$ values. The frequency of 1 $\mathrm{kHz}$, used in the previous work, ${ }^{1}$ gives little indication of an MS relationship. (Note that the data lies out of the range and thus is not shown.)

The solution to all these troubles is the parametric impedance spectroscopy. When the response is explained by the plausible model, including the capacitance parameter $C_{S B}$, a frequency-independent, and uniquely defined $C_{S B}$ can be obtained for each spectrum, or for each potential. It should be noted that CPE was first introduced by Cole and Cole for the non-ideal Debye behavior of the dielectric system, ${ }^{35}$ ) with reference to the ideal $R C$ series circuit, where the capacitance effects are represented by the ideal capacitor and the dispersion is represented by a CPE with $\alpha$ close to 0 limit corresponding to an ideal resistor. Later, the Havriliak-Negami $(\mathrm{HN})$ equation is suggested as a more general description of the dispersive dielectric responses: ${ }^{36}$ )

$$
C_{H N}^{*}=\frac{C_{H N}}{\left(1+\left(j \omega \tau_{H N}\right)^{\gamma}\right)^{\beta}}
$$

With $\gamma=\beta=1$, the equation represents the Debye response, where $\tau=R C$. Note that the Debye circuit is also the model for the relaxation process involving deep trap levels in Schottky diodes. ${ }^{37)}$ The HN equation can thus represent the dispersive, distributed, or non-ideal trapping processes associated with the Schottky junction. Unlike CPE, the equation provides well-defined limiting capacitance magnitude $C_{H N}$, which can be interpreted for physical interpretation as in Eq. (1). Recently, capacitance effects represented by $\mathrm{HN}$ functions connected in parallel have been shown to give more superior descriptions of the impedance behavior of many polycrystalline solid electrolytes. ${ }^{25-28)} \mathrm{Eq}$. (2) is implemented as model DE-31 in a ZView program (Scribner Ass. Inc., USA), as indicated in the equivalent circuit model shown in Fig. 6, center left. In previous publications, ${ }^{26,28) ~ ' ~} \mathrm{H}$ ' symbol was introduced for a simple equivalent circuit diagram. (Note that the model number in the program has been changed from "DE-28 reported earlier. ${ }^{28)}$ ) This is a versatile model, and is expected to play a crucial and essential role in the future impedance spectroscopy.
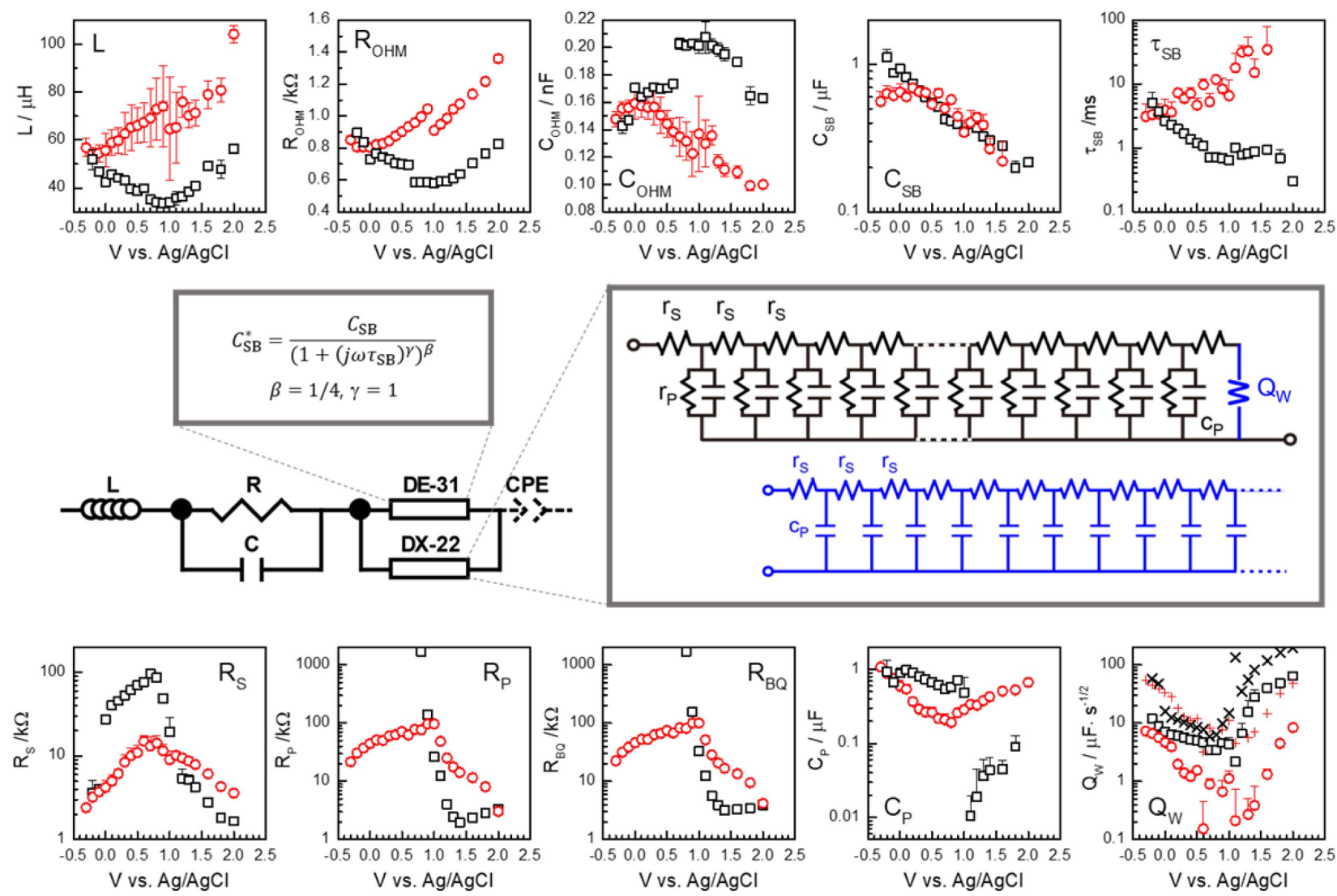

Fig. 6. General impedance model for photoanodes of PEC cells (middle) and the parameters in dark (black squares) and illuminated conditions (red circles) at different potentials. Note that only the upper plus side of the errors is shown, for clarity in the logarithmic plots $\left(R_{S}, R_{P}, R_{B Q}, C_{S B}, \tau_{S B}, C_{P}, Q_{W}\right)$. See the text for details.

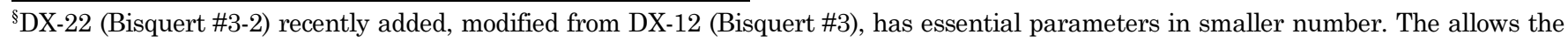
multiparameter, full cell model as shown in Fig. 6 to be run within the 30 parameter limit in the equivalent circuits of the ZView program. 
According to the full parametric analysis, including the Bisquert TL model (DX-22 $2^{\S}$ in Fig. 6) for the diffusionrecombination process as described in the following section, at least for the reverse bias exhibiting the systematic variation as in Fig. 5(b), the power exponents were found almost constant close to $\beta=1 / 4$ with $\gamma=1$, that is:

$$
C_{S B}^{*}=\frac{C_{S B}}{\left(1+j \omega \tau_{S B}\right)^{1 / 4}}
$$

The final fitting presented here was performed with fixed exponents for all spectra. Small variations in the fitted exponents led to strongly correlated variations in the other fitted parameters, so the exponents to be fixed should be determined by preliminary fitting procedures, or by theory if any. ${ }^{25-28)}$
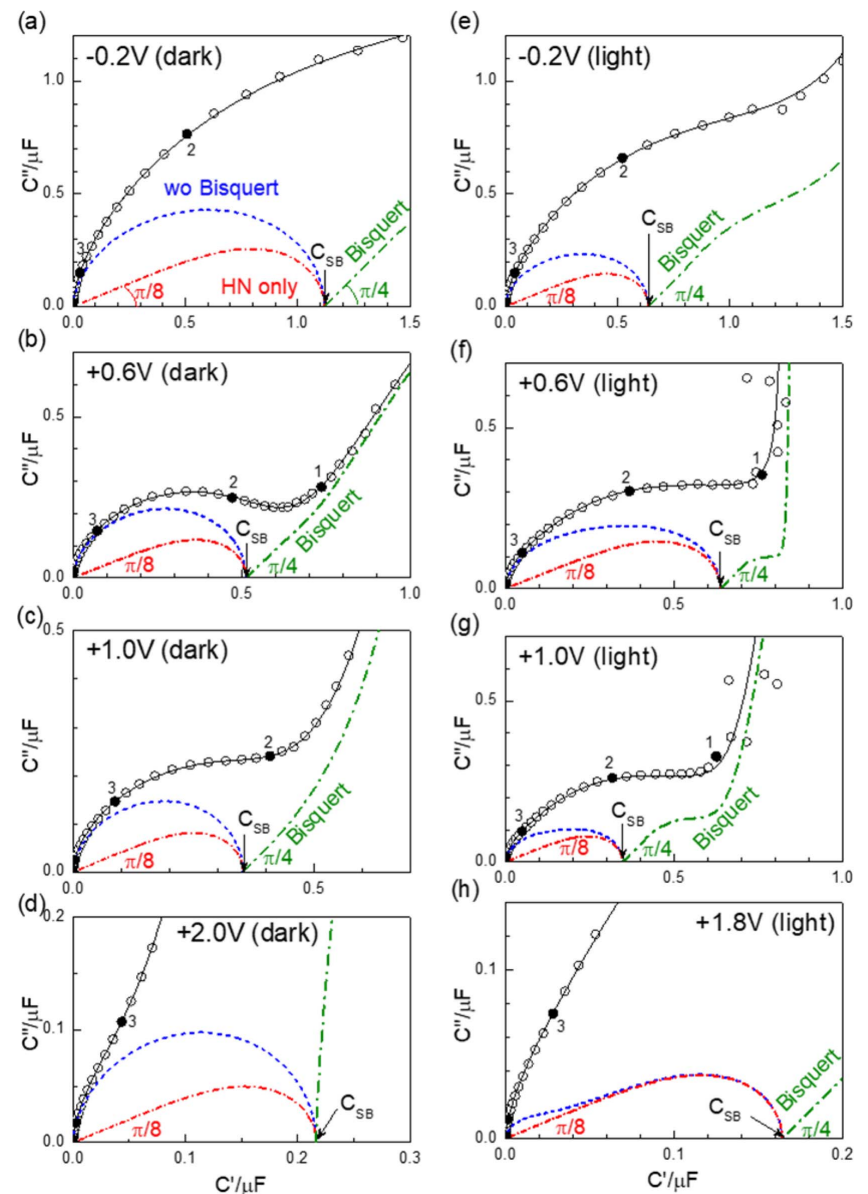

Fig. 7. Selected examples of the capacitance spectra in dark condition ((a)-(d)) and under illumination ((e)-(h)) at different potentials vs. $\mathrm{Ag} / \mathrm{AgCl}$. Simulated HN elements for distributed Schottky capacitance are shown in red dash-dotted lines, with high frequency responses of the phase $\pi / 8$. The dashed blue lines represent the simulation, including the high frequency response described by $L$ and $R C$ parallel circuits, but without the Bisquert TL element (DX-22) (Fig. 6). The Bisquert model in parallel to $C_{S B}$ is shown in green dash-dotted lines. The high frequency response of the constant phase $\pi / 4$ is indicated for the spectrum with finite $C_{P}$.
It should be noted that the apparent capacitance arcs in the experimental spectra of Fig. 5 are largely ascribed to the series $R_{O H M}$ components, as shown by the simulation represented by dashed blue lines in Fig. 7. Separately simulated HN components of Eq. (3) are presented in dash-dotted red lines. At the high frequency range $\omega \rightarrow \infty$, the equation behaves as CPE or $Q$ with $\alpha=3 / 4$, with the constant angle of $(1-\alpha)(\pi / 2)$ or $\pi / 8$. The dispersion in the Schottky capacitance can be attributed to the distribution of donor or trap levels in the $\mathrm{TiO}_{2}$ photoanode, with reference to the case of $\gamma$ $=\beta=1$ for the single trap level. Whether the exponent $\beta=1 /$ 4 should have any mechanistic origin needs further investigation in related and other semiconducting photoanodes or Schottky junctions.

Identification of such components with well-defined power exponents was made possible, since the frequency dispersion parameters for the overlapping Bisquert components, discussed in the next section, are used in the ideal form given by the theoretical model. If multiple frequency dispersion parameters such as $\alpha$ in $\mathrm{CPE}$ or $\gamma$ and $\beta$ in $\mathrm{HN}$ function were left free, there would be little chance to systematically determine model parameters of any physical significance. Frequency power exponents affect the impedance response over the wide frequency range, so it is highly likely that all other fitted parameters would end up strongly correlated with these exponents, just to reach the minimum of the sum of squared errors in the fitting process. This discussion points out the critical problem in the impedance spectroscopy in many different subjects and studies, where modeling with multiple 'magic' CPEs, as $\left(R_{1} Q_{1}\right)\left(R_{2} Q_{2}\right)\left(R_{3} Q_{3}\right)$, allows a very nice description of the experimental spectra, but further significance is barely considered.

The fitted $C_{S B}$ and $\tau_{S B}$ parameters of Eq. (3) are presented in Fig. 6, together with other parameters. The somewhat abrupt change in $C_{S B}$ and $\tau_{S B}$ between $0.6 \mathrm{~V}$ and $0.7 \mathrm{~V}$ in dark condition is in accordance with the behavior of the raw experimental data. The feature is most clearly indicated in the parameters for the high frequency responses $L, R_{\text {Онм }}$, and $C_{\text {ОНM }}$ in Fig. 6. Unlike the continuous voltage scanning of potential for the current or for the monofrequency impedance conventionally done, wide frequency range impedance measurements with stepwise change in the potential, as done in this work, cause some difficulty in obtaining systematically varying reproducible raw data sets. Faradaic currents affect cell behavior during measurements, both temporarily and in the longer term.

Figure 8(a) presents MS plots of parametrically obtained $C_{S B}$. A well-defined linear relation can be found between 0.2 $\mathrm{V}$ and 0.6 V. According to Eq. (1), using the macroscopic electrode area defined by the hole in the quartz cuvette, 7 $\mathrm{mm}^{2}, N_{D}$ and $V_{F B}$ were estimated as $3.2 \times 10^{19} \mathrm{~cm}^{-3}$ and 0.17 $\mathrm{V}$ vs. $\mathrm{Ag} / \mathrm{AgCl}$, respectively. It is notable that a similar MS slope was indicated above $0.7 \mathrm{~V}$. Consistent $N_{D}$ values may be estimated with some difference in the intercept or $V_{F B}$ as $0.35 \mathrm{~V}$. The shift in the intercept, or $V_{F B}$, may be explained by the change in surface polarization state, or the formation 

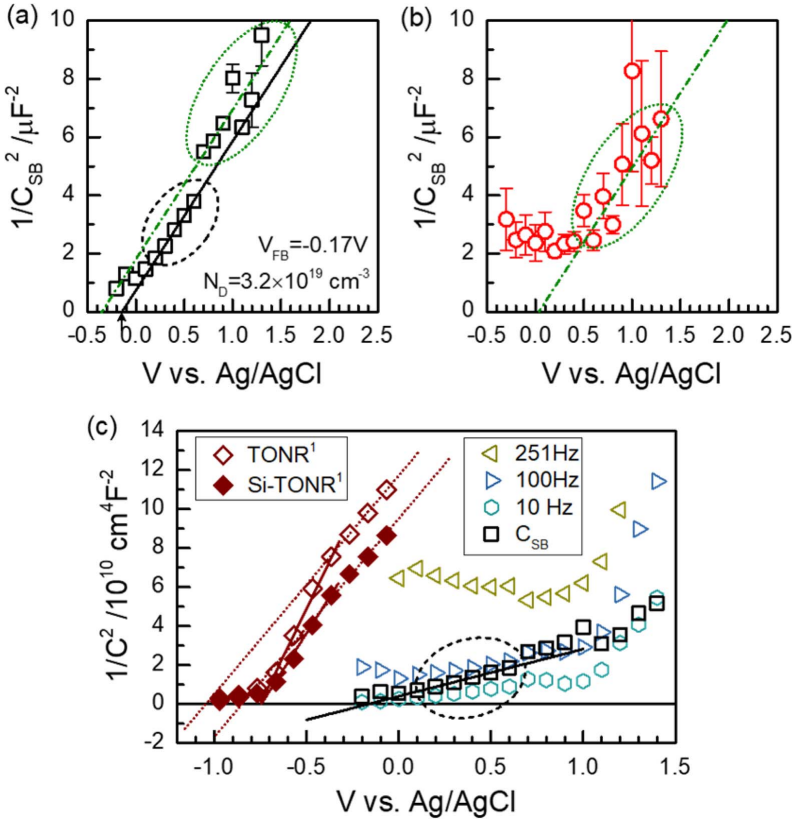

Fig. 8. Mott-Schottky plots of Schottky capacitance $C_{S B}$ under dark (a) and illuminated (b) conditions. In (a) Mott-Schottky analysis is from the data range between $0.2 \mathrm{~V}$ and $0.6 \mathrm{~V}$ vs. $\mathrm{Ag} / \mathrm{AgCl}$. In (c), capacitance values at the frequencies $10 \mathrm{~Hz}, 100 \mathrm{~Hz}$, and $251 \mathrm{~Hz}$ are compared with $C_{S B}$. The results from Ref. 1 are also presented.

of the Helmholtz layer at the solution side of the interface during the measurements. The deviation below $0.2 \mathrm{~V}$ is ascribed to the potential approaching $V_{F B}$ and cathodic range.

In the measurement under illumination, a discontinuity occurred between $0.9 \mathrm{~V}$ and $1.0 \mathrm{~V}$. This is shown in the parameters $L, R_{O H м}$, and $C_{O H м}$, and less clearly in $C_{S B}$ and $\tau_{S B}$, all represented by red symbols in Fig. 6 . The corresponding MS plot is given in Fig. 8(b). The parameters evaluated from the data under illumination are shown associated with large errors, except $R_{\text {ОНм }}$. Note that large statistical errors are generally associated with overparameterization. The broad capacitance response of Fig. 7(e), (f), and (g) under illumination is described by the overlapping responses of an HN function (DE-31), and a Bisquert TL model (DX22 ), as well as of other components for the high frequency response. This is in contrast to the measurement in dark conditions, where the magnitude of $C_{S B}$ is rather directly indicated in the raw capacitance spectra. Moreover, the low frequency measurements under illumination conditions were very poor. Under such limitations, an MS behavior is suggested at high reverse bias above $0.5 \mathrm{~V}$. Using a slope similar to that for dark conditions, $V_{F B}$ may be extrapolated near $0 \mathrm{~V}$. The difference from the dark condition may be qualitatively explained by the decrease in Schottky barrier potential, consequent to the photoinduced charge carriers.

In Fig. 8(c), parametric $C_{S B}$ from Fig. 8(a) and some monofrequency MS plots are compared. The results are in great contrast to the MS plots at $1 \mathrm{kHz}$ reported earlier ${ }^{1)}$ using the $\mathrm{S} 2$ setup in Fig. 1(b) for the pristine and $2 \% \mathrm{Si}$ doped $\mathrm{TiO}_{2}$ nanorods. The AC behavior is completely different from the present work using the $\mathrm{S} 1$ setup, and the substantial differences between the two measurements can be mainly attributed to the setups. Different quartz vessels and different positions of the electrodes, as shown by S1 and S2 in Fig. 1(b), result in different high frequency responses, which is strongly affected by the ohmic contribution. Moreover, the near $V_{F B}$ and cathodic potential are strongly affected by the reference electrodes, R1 and R2, as illustrated in Fig. 2(a). The difference in $V_{F B}$ or OCV is also partly explained by the $\mathrm{pH}$ value, 14 , used in the previous work compared with this work $(\mathrm{pH} 13)$, which increases $V_{F B}$ by $0.059 \mathrm{~V}$ at RT. Fresh $\mathrm{TiO}_{2}$ electrodes exhibit higher $V_{F B}$. Repeated measurements of the samples as in this work, may have changed $N_{D}$, for example by proton uptake by electrochemical doping, ${ }^{9)}$ or the surface chemistry, for example by the redistribution of $\mathrm{Si}$ or any other built-in surface charges even with thorough washing in between.

For the data shown in Fig. 8(c) from the previous report, ${ }^{1)}$ the MS analysis was made for the frequency range around $0.5 \mathrm{~V}$ vs. $\mathrm{Ag} / \mathrm{AgCl}$, indicated in solid lines, which resulted in the conclusion of the same $V_{F B}$ of $0.75 \mathrm{~V}$ vs. $\mathrm{Ag} / \mathrm{AgCl}$ for both samples, i.e. same intercepts and larger $N_{D}$ for Si-doping from the lower slope. The result was supposed to represent the increased electron concentration by Si doping. However, when the linear behavior around $-0.2 \mathrm{~V}$ is considered, similar slopes mean essentially the same $N_{D}$ for both samples, hence, no bulk doping effects. $V_{F B}$ of $1.0 \mathrm{~V}$ for the pristine sample, and $0.85 \mathrm{~V}$ for the Si-doped sample, may be attributed to the $\mathrm{Si}$ segregation on the surface. In fact, Si has negligible solubility in $\mathrm{TiO}_{2}$. The effects on the chemical diffusivity and electrical conductivity in $\mathrm{TiO}_{2}$ containing $\mathrm{SiO}_{2}$ as composites were attributed to the alteration in concentrations of transport-related point defects by the transfer of charged species to internal surfaces. ${ }^{38)}$

The discussion above illustrates the issues associated with the MS analysis, which are the frequency dependence (or dispersion) and potential range dependence, and the consequent controversies in scientific interpretations. The problem with having poorly defined geometry in porous nanocrystalline photoanodes was discussed above using Fig. 4. The $N_{D}$ estimated as $3.2 \times 10^{19} \mathrm{~cm}^{-3}$ according to Eq. (1), using the hole area of $7 \mathrm{~mm}^{2}$ and $\varepsilon_{R} 170$ for $c$-direction, show that $\mathrm{TiO}_{2}$ is highly doped. The $C_{S B}$ of approximately $0.7 \mu \mathrm{F}$ at $0 \mathrm{~V}$ vs. $\mathrm{Ag} / \mathrm{AgCl}$ corresponds to a $15 \mathrm{~nm}$ thickness of the depletion space charge layer. However, if the true surface area of the electrode with $\mathrm{TiO}_{2}$ nanorods becomes 100 times larger, it would reduce the estimation of $N_{D}$ by $10^{4}$ times, i.e. $3.2 \times 10^{15} \mathrm{~cm}^{-3}$, which is very low-doping. The depletion layer thickness becomes $1.5 \mu \mathrm{m}$ and all nanorods would be electron depleted. When the lateral surface of the $c$-axis-oriented $\mathrm{TiO}_{2}$ rods is considered, $\varepsilon_{R}$ in the perpendicular direction 86 should be used, which increases $N_{D}$ twice and decreases the thickness of the depletion layer by half. 

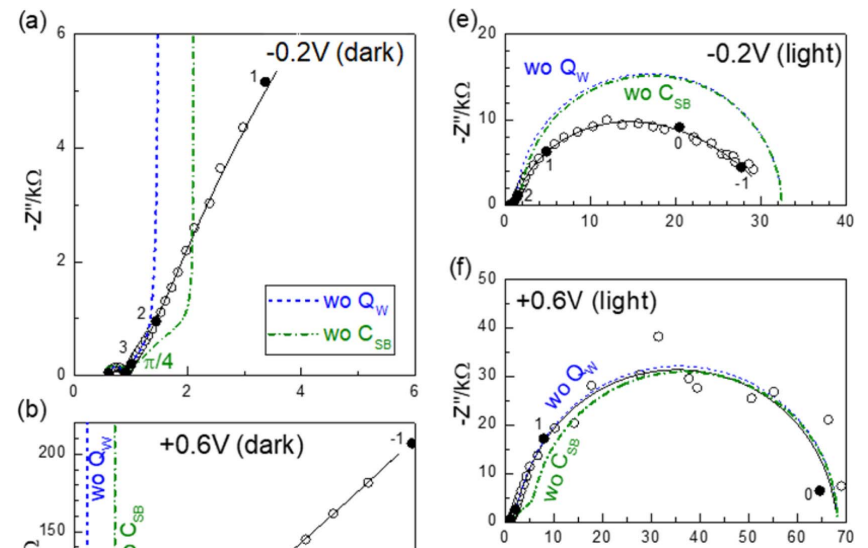

$(\mathrm{g})_{25}$

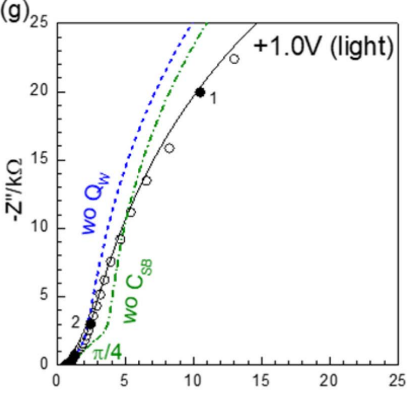

(h)

(d)
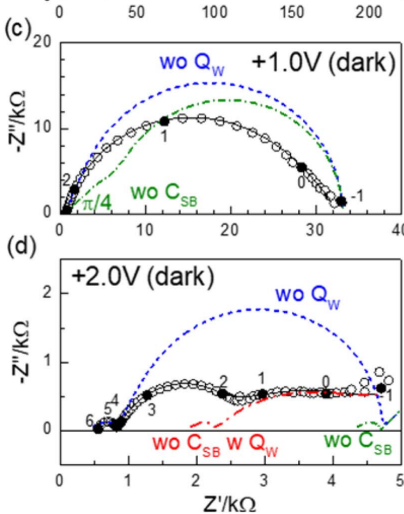

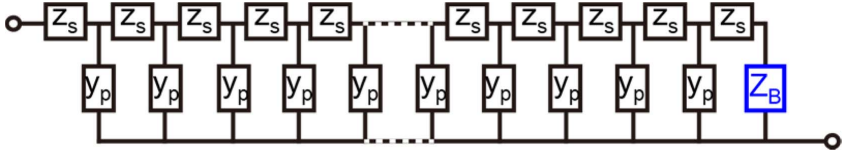

Fig. 10. A general form of Bisquert TL models DX12, 22, 26 , and 27 , with interfacial impedance.

\subsection{Bisquert TL Model with $Z_{B}$}

For a purely semiconducting Schottky diode, the impedance should end up being purely capacitive, as shown by the simulated $\mathrm{HN}$ response in Fig. 7, and possibly with leakage current, represented by a parallel resistor $R_{D C}$, which gives a diverging vertical response, apparently somewhat similar to Fig. 7(f). There are however more involved additional responses, as representatively shown in Fig. 7(b). Fig. 9 shows the impedance plane plots corresponding to the complex capacitance plots in Fig. 7, except for Fig. 7(h). Figure 7(h) shows the response at $1.8 \mathrm{~V}$, while Fig. 9(h) shows the impedance response at $2.0 \mathrm{~V}$. Fig. 9(h) indicates the low frequency feature described by a TL model, characterized by the slope-one, high frequency 'Warburg' impedance discussed below. The spectrum does not allow a deconvolution of the Schottky capacitance component. Two presentations in Fig. 7 and Fig. 9 illustrate that the overall impedance is successfully described by a Bisquert TL model (DX-22) in parallel to the HN capacitance function (DE-31), which is suggested as a physically plausible model for the Schottky contact of a semiconductor with possibly distributed trap levels.

The Bisquert TL model employed in this work can be generally represented as is shown in Fig. 10:39) the longitudinal rail of impedance $Z_{S}=\Sigma z_{s}$, admittance in transverse direction or shunt admittance $Y_{P}=\Sigma y_{p}$, with the connected in series to $Z_{S}$ and in parallel to $Y_{P}$. The interfacial impedance $Z_{B}$ component has a special position in the TL model and cannot be located independently from it. A more generalized model may contain a finite impedance of the bottom rail, with an interfacial impedance $Z_{A}$ connected in series. When the bottom rail is of negligible resistance, $Z_{A}$ can be taken outside of the entire TL model. One type of the carriers is often indeed much more conductive than the other. The impedance of this general TL model is given by the following:

$$
Z^{*}=\frac{1+Z_{B} \sqrt{\frac{Y_{P}}{Z_{S}}} \operatorname{coth} \sqrt{Z_{S} Y_{P}}}{Z_{B} \frac{Y_{P}}{Z_{S}}+\sqrt{\frac{Y_{P}}{Z_{S}}} \operatorname{coth} \sqrt{Z_{S} Y_{P}}}
$$

With $Z_{B} \rightarrow \infty$, it becomes

$$
Z^{*}=\sqrt{\frac{Z_{S}}{Y_{P}}} \operatorname{coth} \sqrt{Z_{S} Y_{P}}
$$

For the electrochemical impedance of the porous electrodes, a simple theoretical model of three parameters $R_{S}, R_{P}$, and that the $L$ and $C_{O H M}$ parameter explain the high frequency behavior in a manner correlated with that of $R_{\text {Онм }}$. 
$C_{P}$, as shown in Fig. 6, can be considered, i.e. $Z_{S}=R_{S}=\Sigma r_{S}$, $Y_{P}=R_{P}^{-1}+j \omega C_{P}=\Sigma r_{P}^{-1}+j \omega \Sigma c_{P}$. The impedance magnitude of the Bisquert TL at $\omega \rightarrow 0$ becomes

$$
R_{B Q}=Z^{*}(\omega \rightarrow 0)=\sqrt{R_{S} R_{P}} \operatorname{coth} \sqrt{\frac{R_{S}}{R_{P}}}
$$

when $Z_{B}(\omega \rightarrow 0) \rightarrow \infty$. It applies to the Warburg impedance of infinite length as $Z_{B}$ in Fig. 6, as well as to the simple open-circuit. For the short-circuited $Z_{B}(\omega \rightarrow 0) \rightarrow 0$, the magnitude becomes less as

$$
R_{B Q}=Z^{*}(\omega \rightarrow 0)=\sqrt{R_{S} R_{P}} \tanh \sqrt{\frac{R_{S}}{R_{P}}}
$$

As discussed by Bisquert et al. ${ }^{8,10)}$ there is a degeneracy of TL models involving transport. As far as the small signal AC impedance is concerned, the model for porous electrodes in which the transport is by drift in the electrical field and the electrochemical reaction, e.g. following a Butler-Volmer relationship, occurs at the distributed interfaces, is equivalent to the diffusion-reaction(recombination) model. The actual physical mechanisms can be distinguished by examination of the behavior of the parameters in different conditions. The diffusion-recombination model has been successfully applied to DSSCs with low-doped nanostructured $\mathrm{TiO}_{2}$ electrodes, where no electrical potential gradient or electron drift is supposed to occur, as the electron charge in the nanostructured $\mathrm{TiO}_{2}$ is compensated by the positive ion charge of the surrounding electrolyte. Diffusion of the electrons and bulk recombination are concerned, as for the electron minority carriers in the $p$-region of the $p$ - $n$ junction. The same model has been applied to the porous gas electrodes of solid oxide fuel cells ${ }^{40)}$ where the (surface) diffusion and adsorption reaction model by Mizusaki can be applied, ${ }^{41)}$ rather than the charge transfer reaction model of traditional electrochemistry. It is also likely that additional TL model components are present such as the diffusion process within the liquid electrolytes in the classical electrochemical cells or DSSCs, ${ }^{4)}$ or gas concentration phenomena in fuel cells. ${ }^{40)}$ The identification of the respective TL components may not be trivial. Some indication of Warburg impedance in the electrolyte is given in Fig. 9(d) and (h), which is represented by a CPE in a dashed line in Fig. 6, with $\alpha=1 / 2$. The bent capacitor symbol or $>>$, originally suggested for the program LEVM by Macdonald, ${ }^{42)}$ is employed in the ZView program.

In the porous electrodes electrical network model, $C_{P}$ represents the double layer capacitance proportional to the charge accumulation and the enlarged interface area of the porous structure. On the other hand, when the diffusion mechanism is involved, $C_{P}$ represents chemical capacitance, $C_{\text {CHEM }}$

$$
C_{C H E M}=\frac{q^{2} n}{k T} G
$$

where $n$ represents the carrier concentration per area or per volume, depending on the mechanism concerned. The geom- etry factor $G$ becomes the surface area or the volume of the electrode material, respectively. The diffusion coefficient in Fick's law (or the chemical diffusivity), reaction rate constant, and the effective reaction zone (or utilization length), can be directly derived by the three parameters of the TL model, $R_{S}, R_{P}$, and $C_{P}$, as

$$
\begin{aligned}
& D_{C H E M}=\frac{L^{2}}{R_{S} C_{P}} \\
& k_{R X N}=\frac{1}{R_{P} C_{P}} \\
& L_{E F F}=\sqrt{\frac{R_{P}}{R_{S}}}
\end{aligned}
$$

(These derived parameters are presented in Fig. 12.) Effective reaction zone is represented by the ratio of $R_{P}$ to $R_{S}$. With large $R_{S}$, i.e. small $D_{\text {CHEM }}$, the process is diffusion limited, and $L_{E F F}<100 \%$, that is, only a part of the electrode thickness is utilized. Large $R_{P}$, that is, small $k_{R X N}$, means small recombination in semiconductor solar cells. Consequent $L_{E F F} \gg 100 \%$ or the entire electrode thickness being effective leads to the high performance for solar cells. On the other hand, both high transport and reaction rates are desirable for the electrochemical conversion. Around 50\% utilization length has been found for SOFC electrodes. ${ }^{40)}$

In the previous section, $\mathrm{HN}$ dielectric function was suggested for the dispersed Schottky capacitance behavior. Unlike the popular CPEs, the model allows the determination of well-defined capacitance parameters, which can be used for MS analysis. Bisquert's group has played an essential and pioneering role in the rigorous derivation of the TL models and in the exhaustive and comprehensive associated physical interpretations. Much emphasis has been given on the capacitance parameters. Similarly, as for the Schottky capacitance, well-defined $C_{P}$ parameters are needed for the straightforward physical interpretations, such as double layer capacitance, or the chemical capacitance in Eq. (8). With $C_{P}$ together with $R_{S}$ and $R_{P}$, the ideal, three-parameter Bisquert model is characteristic for the slope-one, high frequency behavior and semicircular, low frequency end with a vertical slope, as shown by the impedance spectrum in the blue line in Fig. 11(a), labeled as 'wo $Q_{W}$ '. In this case, the capacitance Bode plot should show a plateau as shown in Fig. 11(b). The plateau values approach $C_{P}$ when $R_{P} \gg R_{S}$, or when the low frequency spectrum represents $\left(R_{P} C_{P}\right)$ parallel circuit.

However, most of the real experimental data for the TL modeling cannot be described by the three parameters, $R_{S}$, $R_{P}$, and $C_{P}$. The deviation is accommodated by the 'useful' and 'indispensable' $\mathrm{CPEs},{ }^{4)} C_{Q}^{*}=Q(j \omega)^{\alpha-1}$, instead of $C_{P}$, while the resistance parameters $R_{S}, R_{P}$, are kept regular. The CPE behavior is attributed to the distribution in the chemical capacitance, for example, due to the distributed trap levels in the disordered material in $\mathrm{TiO}_{2}$ nanostructured electrodes in DSSCs. Note that $Q_{P}$ in the TL model represents the deviation of the high frequency slope from 
$\tan (\pi / 4)=1$ as $\tan ((\pi / 4) \alpha)$, and the low-frequency arc as the depressed semicircle of the $\left(R Q_{P}\right)$ parallel connection. ${ }^{43)}$ In Fig. 12, the ideal Bisquert model with $C_{P}$ and $Q_{P}$ with $\alpha=$ 0.8 is compared. The $\alpha$ values as 0.8 are the typical results in the analysis of DSSCs by the Bisquert model. ${ }^{4)}$ Note that $Q_{P}$ in Bisquert TL model results in correlated high and low frequency behavior. Such skewed impedance arcs have been described by an $\mathrm{HN}$ equation in impedance form, ${ }^{44)}$ i.e.,

$$
Z_{H N}^{*}=\frac{R_{H N}}{\left(1+\left(j \omega \tau_{H N}\right)^{\gamma}\right)^{\beta}}
$$

which is probably more known and practiced in the impedance analysis ${ }^{45,46)}$ than the original HN dielectric function. The model has been long since provided in the CNLS (now LEVM) program by Macdonald, and as the DE-6 model in ZView. Empirically, there has been little indication of closely correlated high frequency and low frequency dispersion behavior as required in Bisquert TL model with CPE.

As is in $(R Q)$ modeling, the question arises how the capacitance magnitude, represented by Eq. (8), needed for the derivation of the kinetic parameters $D_{C H E M}$ and $k_{R X N}$ according to Eqs. (9) and (10), can be determined. As discussed above, the effective capacitance ' $C$ ' is defined by the peak frequency or time constant $(R Q)^{1 / \alpha}$ for the $(R Q)$ parallel circuits as $\left(R^{\prime} C^{\prime}\right)$. Note that capacitance values are determined by the resistance parameters. Such correlation may not be rigorously justified. Moreover, for the TL model with two resistor components, $R_{S}$ and $R_{P}$, it is not clear which resistor component should be used. The $R_{S} Q_{P}$ TL represents the diffusion mechanism, so the diffusivity for the anomalous transport or fractal diffusion is estimated from the relaxation time $\tau_{d}=\left(R_{P} Q_{P}\right)^{1 / \alpha}$, while the recombination (reaction) rate is estimated from the time constant $\tau_{\text {rec }}=\left(R_{P} Q_{P}\right)^{1 / \alpha}$. The feature of $Q_{P}$ modeling is illustrated in Fig. 11. Two $Q_{P}$ simulations are distinguished in the Bode plots (not

(a)

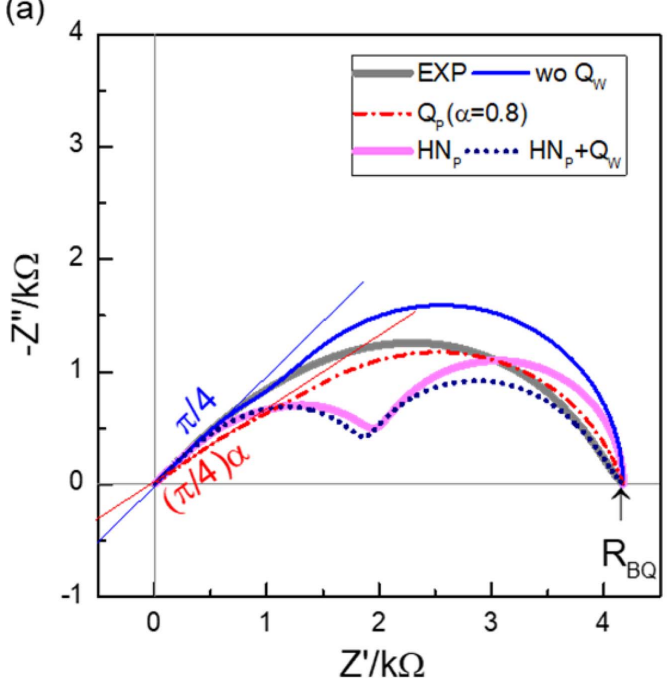

in the impedance spectra). With the $Q_{P}$ coefficient in $C_{Q}^{*}=Q(j \omega)^{\alpha-1}$ of the same value as that of $C_{P}$, the capacitance magnitude becomes smaller when $\alpha$ is less than 1 . Modeling with the $Q_{P}$ element of the experimental data would give a larger value for the $Q_{P}$ parameter, as shown by the simulation at 10 times larger. The sloped behavior at low frequencies is characteristic of $Q_{P}$, and the characteristic inflection points depend on the associated resistance parameters. Therefore, it cannot be stressed too much that there is no rigorous way to determine the capacitance values from the modeling using CPEs.

This issue was pointed out in the application of the Bisquert TL model for fuel cell electrodes ${ }^{40,47,48)}$ and the Jamnik-Maier model for mixed conductors. ${ }^{49-51)}$ With these issues taken into consideration, the electrode responses of full, high temperature fuel cells were described by four TL models in the ideal form with three parameters of $R_{S}, R_{P}$, and $C_{P}$, which represented electrochemical polarization and gas concentration impedance for the anode and cathode, respectively. ${ }^{40)}$ The apparent success in reasonably describing the spectra and systematically obtaining the parameters may be due to the strongly overlapped four TL responses. The strongly depressed semicircular responses for the symmetrical cells theoretically containing only two TL elements were difficult to describe with the Bisquert TL model with ideal capacitance parameter, $C_{P .}{ }^{48)}$ In order to compare two different current collectors the frequency exponent $\alpha$ of $Q_{P}$, was fixed to $0.5 .^{48)}$ The most obvious serious trouble with CPE modeling is that the other circuit parameters, as well as the coefficient $Q$ in $C_{Q}^{*}=Q(j \omega)^{\alpha-1}$, vary in strong correlation with $\alpha$, which is adjusted arbitrarily for the highest overall best fit of the spectra.

This work suggests a more powerful and essential modification of the Bisquert TL model to be widely applicable to experimental data, and also providing clear physical inter-

(b)

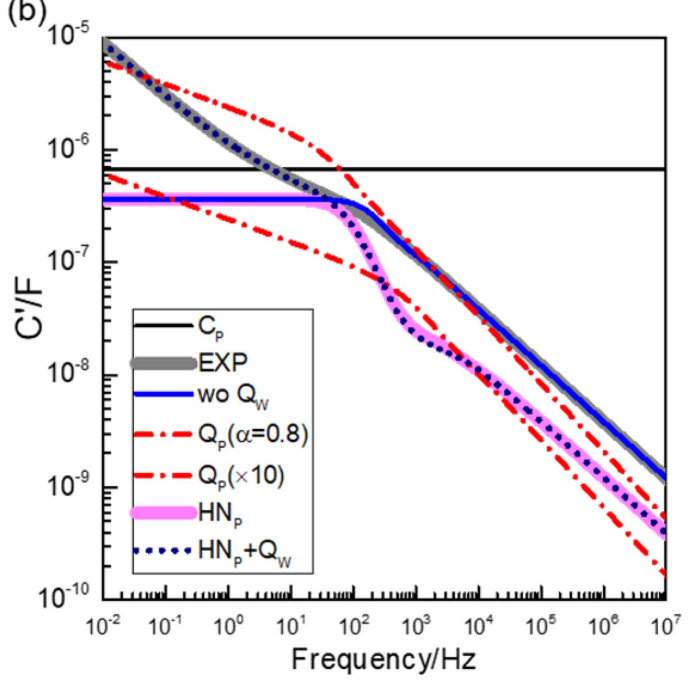

Fig. 11. Simulation of the Bisquert element of Fig. 9(h) (EXP): $R_{S}=3606 \Omega, R_{P}=3054 \Omega, C_{P}=0.6686 \mu \mathrm{F}, Q_{W}=8.3775 \mu \mathrm{F} \cdot \mathrm{s}^{1 / 2}$. Simulations without $Q_{W}$ element, with $Q_{P}=0.6686 \mu \mathrm{F} \cdot \mathrm{s}^{0.2}$ and $6.686 \mu \mathrm{F} \cdot \mathrm{s}^{0.2}(\alpha=0.8)$, and with HN capacitance of $C_{H N}=$ $0.6 \mu \mathrm{F}, \tau=0.001 \mathrm{~s}$, and $\gamma=\beta=1$ as shunt capacitance, with and without $Q_{W}$, are compared in impedance plane (a) and capacitance Bode plot (b). 
pretations. As indicated in the spectrum of Fig. 9(h), slopeone behavior is often clearly indicated in the high frequency region, which is not consistent with the depressed arc behavior of the low frequency region according to Bisquert TL model with $Q_{P}$. Slope-one behavior is a clear indication of the ideal form of shunt capacitance $C_{P}$, which is also desirable for the unambiguous physical interpretation of $D_{C H E M}$ and $k_{R X N}$. It turned out that such inconsistency in high and low frequency behavior could be successfully described by the presence of infinite length $R C$ TL or Warburg impedance as the interfacial impedance $Z_{B}$ in Fig. 10, as shown in the equivalent circuit of Fig. 6 . The modeling was motivated by the indication of the universal presence of the ideal Warburg electrode response in the impedance of polycrystalline solid electrolytes. ${ }^{25,27,28)}$ Note that the infinite length $R C$ TL for $Z_{B}$ requires only one parameter, since the impedance of infinite TL is $Z^{*}=\sqrt{Z_{S} / Y_{P}}=\sqrt{R /(j \omega C)}=1 / Q_{W}(j \omega)^{1 / 2}$, i.e. $Q$ element with $\alpha=1 / 2$ as $C^{*}=Q_{W}(j \omega)^{-1 / 2}$ where $Q_{W}=$ $\sqrt{C / R}$. Independent determination of the $R$ and $C$ parameters is not possible. So the TL model developed in this work contains 4 parameters: $R_{S}, R_{P}, C_{P}$, and $Q_{W}$. The full model, including such TL with 4 parameters in parallel to $\mathrm{HN}$ capacitance function with 2 parameters (since the exponents are fixed) as shown in Fig. 6, can be successfully applied to the impedance data at all potentials, both in dark and illumination conditions.

The impedance spectrum of Fig. 9(h) represents the TL model response directly, as the HN capacitance component cannot be reliably separated, i.e., the spectrum does not need that component for the description. That TL response of the 4 fit parameters $R_{S}, R_{P}, C_{P}$, and $Q_{W}$ is shown in Fig. 11, labeled as EXP. All the spectra in Fig. 11 use the same $R_{S}$ and $R_{P}$. In Bode plots of Fig. 11(b), the effect of the $Q_{W}$ element is clearly shown. While the response without $Q_{W}$, the blue line labeled "wo $Q_{W}$ ", indicates a well-defined capacitance plateau at low frequencies, $Q_{W}$ adds the sloped behavior close to $-1 / 2$. The capacitance effects are additive, as can also be seen in the equivalent circuit of Fig. 6 . The high frequency response is shown to be essentially described by $R_{S}, R_{P}$ and $C_{P}$, regardless of $Z_{B}$. Note that for any $Q_{P}$ with $\alpha<1$ as $Z_{B}$, or as long as no shunt conductance or interface 'leakage' exists, i.e. $Z_{B}(\omega \rightarrow 0) \rightarrow \infty$, the magnitude of TL is determined by $R_{S}$ and $R_{P}$ as $\sqrt{R_{S} R_{P}} \operatorname{coth} \sqrt{R_{S} / R_{P}}$ (Eq. (6)).

Similarly as discussed above for the Schottky capacitance, the HN capacitance function with well-defined, low-frequency limiting magnitude can be a generalized $C_{P}$. With $\gamma$ $=\beta=1$, the model represents a trapping process described by $R C$ series circuit. Without $R_{P}$ (or $R_{p} \rightarrow \infty$ ) this corresponds to the diffusion-trapping process, one of the diffusion-related processes considered by Bisquert.6) Generally, the model contains parameters for drift, diffusion, (generation) recombination, and trapping processes much discussed in semiconductor device physics. ${ }^{14,15)}$ Note that with the same $R_{S}$ and $R_{P}$, the capacitance distribution, for example due to trapping, leads to well-distinguished two arcs, as shown in Fig. 11, for $\tau=0.001 \mathrm{~s}$ and $C_{P}$ divided into an $\mathrm{HN}$ contribution of $0.6 \mu \mathrm{F}$. Modified Bisquert models with an $\mathrm{HN}$ function as a general shunt capacitance, are newly available as DX-25, 26, and 27, in ZView software program (version 3.5e). The additional effect of $Q_{W}$ depressing the semicircular responses is also presented. Note that such spectral features are generally described by $\left(R_{1} Q_{1}\right)\left(R_{2} Q_{2}\right)$, and it is attempted to separate two resistance components $R_{1}$ and $R_{2}$ constituting the total resistance as $R_{1}+R_{2}$. In contrast, the overall resistance of TL containing two resistance components, $R_{S}$ and $R_{P}$, is represented by Eq. (6). It may be noted that the distributed $C_{P}$ and the interfacial capacitance are all additive. This is also in line with the recent suggestion of the superiority of additive capacitance effects in parallel network in describing polycrystalline solid electrolytes to the conventional additive resistance effects in series network, well known as brick-layer model. ${ }^{25-28)}$

\subsection{TL Mechanisms}

When the polarization resistance $R_{B Q}$ is small at high reverse bias close to $2 \mathrm{~V}$, as shown in Figs. 9(d) and (h), the presence of additional low frequency Warburg impedance is indicated. In Fig. 6, the component is shown by the dotted CPE $Q$ element, with $\alpha=1 / 2$ in series to the parallel connection of DE-31 and DX-22. The component represents $R C$ TL. (It should be distinguished from $Z_{B}$ within Bisquert TL (DX22).) The response is attributed to the mass concentration
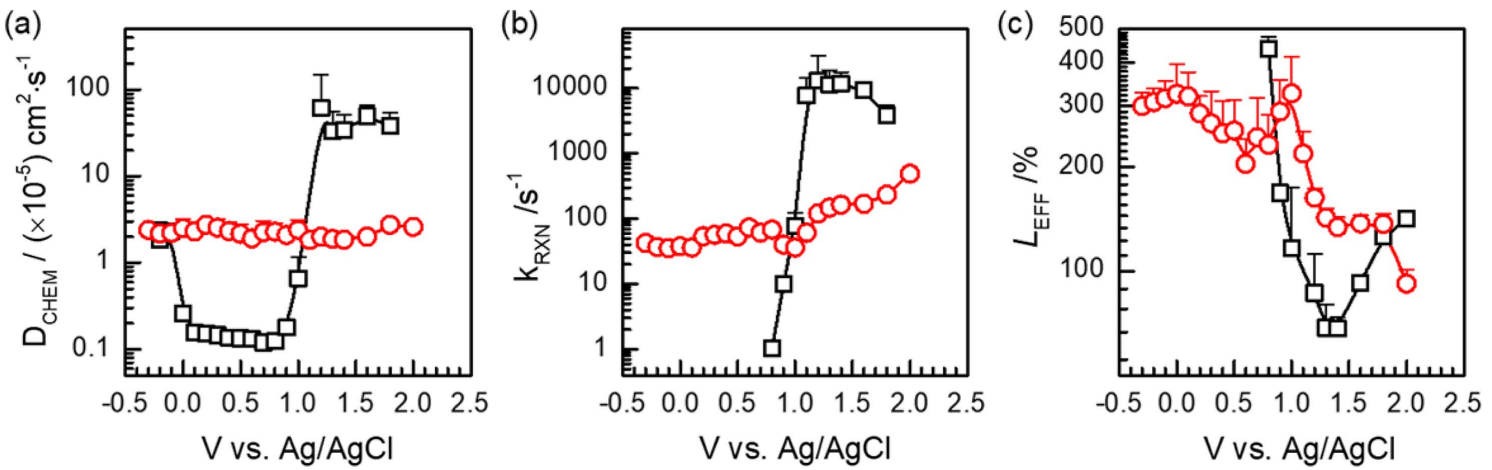

Fig. 12. Chemical diffusivities (a), reaction rates (b), and effective thickness in percent (c), derived from the Bisquert parameters of $R_{S}, R_{P}$, and $C_{P}$. 
impedance in the electrolyte solution, present in all electrochemical cells that have liquid electrolytes. The component is not affected by the potentials or illumination, but can be observed when the electrode polarization resistance becomes small enough.

As discussed previously, the origin of Bisquert TL (DX-22) in parallel to Schottky capacitance, represented by $\mathrm{HN}$ dielectric function (DE-31), should be considered two-fold: drift or diffusion; charge transport in the electrochemical network of porous electrodes, or by the diffusion-recombination mechanism of electrons in $\mathrm{TiO}_{2}$, represented by Eqs. (8)-(10), following the mechanism in DSSCs. In the latter, no electrostatic potential gradient within the low-doped nanostructured $\mathrm{TiO}_{2}$ electrode layer is assumed. The mechanism also requires a particle size of a few tens of nanometers, so that the charges are compensated by ionic charge at the $\mathrm{TiO}_{2} /$ electrolyte interface. The condition seems not to be met in the case for $\mathrm{TiO}_{2}$ nanorods of the microstructure schematically shown in Fig. 4 and also due to the presence of Mott-Schottky behavior shown in Fig. 8.

According to the diffusion-recombination model applied to nanostructured $\mathrm{TiO}_{2}$ electrodes in DSSCs, donor concentration $n$ of the low doped $\mathrm{TiO}_{2}$ depends exponentially on the applied (reverse) voltage as $\exp (-q V / k T)$, since the applied voltage can homogeneously change the Fermi level of the nanostructured $\mathrm{TiO}_{2}$, while the charge neutrality is maintained by the positive ion charge at the semiconductor/electrolyte interface. ${ }^{10)}$ According to Eq. (8), $C_{P}$, being proportional to $n$, should have the same dependence. In the simple approximation, the transport resistance, $R_{S}$, inversely proportional to the conductivity, and the recombination resistance, $R_{P}$, inversely proportional to the electron concentration, they are all proportional to $\exp (+q V / k T)$. It corresponds to the very large negative or positive slope of 17 in the logarithmic values of the parameters vs. $V$ at room temperature. In Fig. 6 , the correct sign but much weaker dependence $(<1)$ is indicated in the parameters $R_{S}, R_{P}$, and $C_{P}$, at the potential less than $0.7 \mathrm{~V} \mathrm{vs}$. $\mathrm{Ag} / \mathrm{AgCl}$. The observation is quantitatively comparable to the behaviors of the aged DSSCs, or DSSCs with highly doped $\mathrm{TiO}_{2} \cdot{ }^{9}{ }^{9}$ Fresh DSSCs exhibited stronger dependence, but still less than the theoretical value. The aged behavior was attributed to the doping during the operation. It is notable that the opposite variations in $R_{S}, R_{P}$, and $C_{P}$, occur above $0.7 \sim 1.0 \mathrm{~V} \mathrm{vs.} \mathrm{Ag} /$ $\mathrm{AgCl}$, where the leakage or breakdown becomes predominant upon reverse bias. The dependence is shown to be more or less symmetrical under the illumination condition (red circles), while abrupt change occurs in the dark condition (black squares).

Figure 12 shows that the diffusivity $D_{C H E M}$ in the dark condition becomes almost constant and small between -0.1 $\mathrm{V}$ to $0.9 \mathrm{~V}$ in the reverse bias region, and a stepwise increase by 500 times above $1.2 \mathrm{~V}$. The dependence originates from $R_{S}$ and $C_{P}$, which are high in the region of forward bias direction and low in the high reverse bias. Reaction rate constant $k_{R X N}$ behaves similarly. In the for- ward bias region, $k_{R X N}$ is not obtained since $R_{P}$ is too large to be determined. On the other hand, $D_{C H E M}$ becomes independent of the potential under illumination. $D_{C H E M}$ is also constant in the respective potential ranges under the dark condition, i.e. $\mathrm{V}<0.9 \mathrm{~V}$ and $\mathrm{V}>1.2 \mathrm{~V}$. That means the characteristic variations of $R_{S}$ and $C_{P}$ with potential are exactly opposite in logarithmic scale. This is because both $R_{S}^{-1}$ or conductivity and $C_{P}$ in Eq. (8) are proportional to the carrier concentration, $n$. A similar situation is observed in the chemical diffusivity of semiconducting oxides little dependent on temperature or atmosphere, since the conductivity and the chemical capacitance are proportional to the charge carrier concentration or to their non-stoichiometry. ${ }^{52)}$

The characteristic $D_{\text {CHEM }}$ behavior under illumination suggests an almost symmetric $n-p$ transition. The electron and hole concentration follow exponential dependence of potential, which is related to the Fermi potential of electrons and holes, $E_{\mathrm{Fn}}, E_{\mathrm{Fp}}$, respectively. Importantly, the variation of the carrier concentrations under illumination is supposed to concern the $\mathrm{TiO}_{2}$ volume responsible for the observed resistance and capacitance values. On the other hand, the absolute values of $R_{S}, R_{P}$, and $C_{P}$, in the dark condition above $1 \mathrm{~V}$ are considered to be affected by the inhomogeneous conduction pathways of breakdown spots, as also indicated by currents not scaled with the area as discussed earlier for Fig. 2. The leakage or breakdown behavior under high reverse bias are strongly affected by the film quality and the state of thesubstrate/ $\mathrm{TiO}_{2}$ interface.

In conclusion, the Bisquert TL parameters qualitatively indicate the diffusion-recombination mechanisms of electronic charge carriers in $\mathrm{TiO}_{2}$. Combination of Schottky capacitance and diffusion-recombination response may not necessarily be contradictory or incompatible. Note that these mechanisms work together in semiconducting structures with depletion of majority carriers at the junction and with the transport of the minority carriers in the bulk. ${ }^{14,15,16)}$ Nanostructured $\mathrm{TiO}_{2}$ and $\mathrm{ZnO}$ electrodes show diffusionrecombination processes in the forward bias region in the aqueous solution used for photoanodes of PEC cells, as well as in the organic electrolyte for the DSSC application. ${ }^{13,53,54)}$ On the other hand, MS analysis is routinely performed in the reverse bias region for identical or similar nanostructured electrodes. There is no abrupt boundary between high and low doping cases, or between meso- or macroscale and nanoscale. Spatial inhomogeneity in the electrode responses may also exist, e.g. at top vs. lateral surface vs. inner volume of the $\mathrm{TiO}_{2}$ nanorods, due to the crystal anisotropy, surface chemistry and structure and/or inhomogeneous doping concentration. Therefore, it may be concluded that, due to the inhomogeneity in the material, or in the potential distribution, and/or in the case of moderate doping level, the two mechanisms are generally coexistent, as represented by the master equivalent circuit in Fig. 6 for semiconducting photoanodes of PEC cells.

The presence of interfacial Warburg impedance as $Z_{B}$ is the important modification of Bisquert TL model for a wide 
applicability. The behavior of $Q_{W}$ parameter in Fig. 6 suggests a very close relationship to the bulk diffusion mechanism, as the estimation of $\sqrt{C_{P} / R_{S}}$ is shown to be similar under the dark and the illumination condition (in black crosses and red plus symbols respectively). This is notable since the individual parameters exhibit non-trivial dependence on the potential and illumination condition. As also suggested by close to half of the activation energy of the Warburg element with respect to the bulk conductivity of the solid electrolytes, ${ }^{25,27)} R$ and $C$ of $Z_{B}$ appear to be closely related to the bulk TL parameters of $R_{S}$ and $C_{P}$ distributed in the 'bulk' of the electrode representing the bulk diffusion mechanism. The present modification is suggested to be a more proper and general TL model of the diffusion-reaction process taking place in many electrochemical systems. The application of the process to solid oxide fuel cell electrodes, ${ }^{48)}$ water oxidation catalysts ${ }^{55)}$ and other systems is under examination.

Although the ideal $C_{P}$ parameter is used in the description of all spectra, the characteristic slope-one behavior can be directly found in only a few spectra at high reverse bias, e.g. Figs. 9(d) and (h). There are three factors to be considered for such a feature. First, when $R_{S}$ is much larger than $R_{P}$, or $D_{C H E M}$ is much smaller than $k_{R X N}$, the slope-one behavior becomes dominant in the TL model response. This diffusionlimited response corresponds to the effective reaction zone or to the utilization length of substantially less than $100 \%$. Secondly, slope-one behavior can be identified in the small portion at the high frequency range, which is likely to be overlapped with other high frequency components such as $R_{\text {Онм }}$ and $L$ and other stray impedance. Thirdly, often the high frequency slopes greater than one results from the overlapping capacitance effects. In the present work the deviation is explained by the Schottky capacitance connected in parallel. Similarly, it has been pointed out that the high-frequency feature of the Warburg impedance of the Hebb-Wagner polarization cells is affected by the double layer capacitance due to the blocked species which is positioned similarly as $Q_{W .}{ }^{49,50)}$ The manifestation of diffusion components in the spectra in Figs. 9(d) and (h) can be ascribed to the negligible contribution of Schottky capacitance at high reverse bias, according to MS behavior. It should be noted that the two capacitance parameters representing each mechanism, $C_{S B}$ and $C_{P}$, are comparable in magnitude, but the respective characteristic potential dependences are well presented. The results can be considered to indicate the successful separation of the two impedance mechanisms.

\section{Conclusions}

Full parametric impedance analysis of the AC response of a $\mathrm{TiO}_{2}$ photoanode in a PEC cell, as a function of the potential and with varied illumination condition, has been performed. Essentially, two capacitance components are connected in parallel: depletion capacitance at the semicon- ductor-electrolyte junction following an MS relationship, and chemical capacitance in the TL model for the electron diffusion-recombination kinetics in the bulk $\mathrm{TiO}_{2}$. Welldefined capacitance parameters can be found by employing an $\mathrm{HN}$ capacitance function, which represents the distributed trap levels of a finite concentration. The Bisquert diffusion-recombination TL model can be applied with chemical capacitance in ideal form, not as CPE, allowing a straightforward interpretation of the diffusivity and recombination rate constant. Interfacial Warburg impedance, closely related to the bulk diffusion mechanism, successfully explains the depressed semicircular responses of the experimental data. The 4-parameter Bisquert TL model is suggested as being generic and widely applicable.

\section{Acknowledgments}

This research was supported by Basic Science Research Program through the National Research Foundation of Korea (NRF) funded by the Ministry of Education (2017R1D1A1B03033918) and by the National Research Foundation of Korea (NRF) grant funded by the Ministry of Science, ICT \& Future Planning (NRF2014R1A2A2A04004950).

\section{REFERENCES}

1. H. S. Kim, D. T. Nguyen, E.-C. Shin, J.-S. Lee, S. K. Lee, K.-S. Ahn, and S. H. Kang, "Bifunctional Doping Effect on the $\mathrm{TiO}_{2}$ Nanowires for Photoelectrochemical Water Splitting," Electrochim. Acta, 114 159-64 (2013).

2. J. Nowotny, Oxide Semiconductors for Solar Energy Conversion: Titanium Dioxide; pp. 364, CRC Press, Boca Raton, 2011.

3. P. Blood and J. W. Orton, The Electrical Characterization of Semiconductors: Majority Carriers and Electron States; Academic Press, New York, 1992.

4. J. Bisquert and F. Fabregat-Santiago, Impedance Spectroscopy: a General Introduction and Application to DyeSensitized Solar Cells; CRC Press, Boca Raton, 2010.

5. F. Fabregat-Santiago, G. Garcia-Belmonte, I. Mora-Sero, and J. Bisquert, "Characterization of Nanostructured Hybrid and Organic Solar Cells by Impedance Spectroscopy," Phys. Chem. Chem. Phys., 13 [20] 9083-118 (2011).

6. J. Bisquert, "Beyond the Quasistatic Approximation: Impedance and Capacitance of an Exponential Distribution of Traps," Phys. Rev. B, 77 [23] 235203 (2008).

7. J. Bisquert, "Theory of the Impedance of Electron Diffusion and Recombination in a Thin Layer," J. Phys. Chem. $B$, 106 [2] 325-33 (2002).

8. J. Bisquert, "Comment on Diffusion Impedance and Space Charge Capacitance in the Nanoporous Dye-Sensitized Electrochemical Solar Cell" and "Electronic Transport in Dye-Sensitized Nanoporous $\mathrm{TiO}_{2}$ Solar Cells Comparison of Electrolyte and Solid-State Devices," J. Phys. Chem. B, 107 [48] 13541-43 (2003).

9. F. Fabregat-Santiago, E. M. Barea, J. Bisquert, G. K. Mor, K. Shankar, and C. A. Grimes, "High Carrier Den- 
sity and Capacitance in $\mathrm{TiO}_{2}$ Nanotube Arrays Induced by Electrochemical Doping," J. Am. Chem. Soc., 130 1131216 (2008).

10. F. Fabregat-Santiago, G. Garcia-Belmonte, J. Bisquert, A. Zaban, and P. Salvador, "Decoupling of Transport, Charge Storage, and Interfacial Charge Transfer in the Nanocrystalline $\mathrm{TiO}_{2} /$ Electrolyte System by Impedance Methods," J. Phys. Chem. B, 106 [2] 334-39 (2002).

11. M. G. Lee and H. W. Jang, "Photoactivities of Nanostructured $\alpha-\mathrm{Fe}_{2} \mathrm{O}_{3}$ Anodes Prepared by Pulsed Electrodeposition," J. Korean Ceram. Soc., 53 [4] 400-5 (2016).

12. S. Kang, R. C. Pawar, T. J. Park, J. G. Kim, S.-H. Ahn, and C. S. Lee, "Minimization of Recombination Losses in 3D Nanostructured $\mathrm{TiO}_{2}$ Coated with Few Layered $\mathrm{gC}_{3} \mathrm{~N}_{4}$ for Extended Photo-response," J. Korean Ceram. Soc., 53 [4] 393-99 (2016).

13. D.-T. Nguyen, E.-C. Shin, D.-C. Cho, K.-W. Chae, and J.S. Lee, "Photoelectrochemical performance of $\mathrm{ZnO}$ Thin Film Anodes Prepared by Solution Method," Int. J. Hydrogen Energy, 39 [35] 20764-70 (2014).

14. C.-T. Sah, Fundamentals of Solid State Electronics; World Scientific Publishing Co. Inc, Singapore, 1991.

15. C.-T. Sah, "The Equivalent Circuit Model in Solid-State Electronics III: Conduction and Displacement Currents," Solid-State Electron., 13 [12] 1547-75 (1970).

16. G. Garcia-Belmonte, A. Munar, E. M. Barea, J. Bisquert, I. Ugarte, and R. Pacios, "Charge Carrier Mobility and Lifetime of Organic Bulk Heterojunctions Analyzed by Impedance Spectroscopy,” Org. Electron., 9 [5] 847-51 (2008).

17. R. de Levie, "On Porous Electrodes in Electrolyte Solutions: I. Capacitance Effects," Electrochim. Acta, 8 [10] 751-80 (1963).

18. E.-C. Shin, H.-H. Seo, J.-H. Kim, P.-A. Ahn, S. M. Park, Y. W. Lim, S.-J. Kim, C. H. Kim, D. J. Kim, C. K. Hong, G. Seo, and J.-S. Lee, "A New Diagnostic Tool for the Percolating Carbon Network in the Polymer Matrix," Polymer, 54 [3] 999-1003 (2013).

19. A. Fujishima and K. Honda, "Electrochemical Photolysis of Water at a Semiconductor Electrode," Nature, 238 [5358] 37-8 (1972).

20. J.-S. Lee and J. Maier, "High Barrier Effects of (0001) | (000-1) Zinc Oxide Bicrystals: Implication for Varistor Ceramics with Inversion Boundaries," J. Mater. Res., 20 [8] 2101-9 (2005).

21. Z. Li, C. Yao, Y. Yu, Z. Cai, and X. Wang, "Highly Efficient Capillary Photoelectrochemical Water Splitting Using Cellulose Nanofiber Templated $\mathrm{TiO}_{2}$ Photoanodes," Adv. Mater., 26 [14] 2262-67 (2014).

22. F. L. Formal, N. Tétreault, M. Cornuz, T. Moehl, M. Grätzel, and K. Sivula, "Passivating Surface States on Water Splitting Hematite Photoanodes with Alumina Overlayers," Chem. Sci., 2 [4] 737-43 (2011).

23. R. A. Parker, "Static Dielectric Constant of Rutile $\left(\mathrm{TiO}_{2}\right)$, 1.6-1060 K," Phys. Rev., 124 [6] 1719-22 (1961).

24. C. Wang, N. Zhang, Q. Li, Y. Yu, J. Zhang, Y. Li, and H. Wang, "Dielectric Relaxations in Rutile $\mathrm{TiO}_{2}, " J . A m$. Ceram. Soc., 98 [1] 148-53 (2015).
25. J.-H. Kim, E.-C. Shin, D.-C. Cho, S. Kim, S. Lim, K. Yang, J. Beum, J. Kim, S. Yamaguchi, and J.-S. Lee, "Electrical Characterization of Polycrystalline Sodium $\beta$-Alumina: Revisited and Resolved," Solid State Ionics, 264 22-35 (2014).

26. S.-H. Moon, Y. H. Kim, D.-C. Cho, E.-C. Shin, D. Lee, W. B. Im, and J.-S. Lee, "Sodium Ion Transport in Polymorphic Scandium NASICON Analog $\mathrm{Na}_{3} \mathrm{Sc}_{2}\left(\mathrm{PO}_{4}\right)_{3}$ with New Dielectric Spectroscopy Approach for Current-Constriction Effects," Solid State Ionics, 289 55-71 (2016).

27. S.-H. Moon, D.-C. Cho, D. T. Nguyen, E.-C. Shin, and J.-S. Lee, "A Comprehensive Treatment of Universal Dispersive Frequency Responses in Solid Electrolytes by Immittance Spectroscopy: Low Temperature AgI Case," J. Solid State Electrochem., 19 [8] 2457-64 (2015).

28. J.-S. Lee, "A Superior Description of AC Behavior in Polycrystalline Solid Electrolytes with Current-Constriction Effects,” J. Korean Ceram. Soc., 53 [2] 150-61 (2016).

29. I. Cesar, K. Sivula, A. Kay, R. Zboril, and M. Grätzel, "Influence of Feature Size, Film Thickness, and Silicon Doping on the Performance of Nanostructured Hematite Photoanodes for Solar Water Splitting," J. Phys. Chem. C, 113 [2] 772-82 (2008).

30. G. Nogami, "Theory of Capacitance-Voltage Characteristics of Semiconductor Electrodes with Interface States," J. Electrochem. Soc., 133 [3] 525-31 (1986).

31. M. Madou, F. Cardon, and W. Gomes, "Impedance Measurements at the N-and P-Type GaP Single Crystal Electrode," J. Electrochem. Soc., 124 [10] 1623-27 (1977).

32. B. Klahr, S. Gimenez, F. Fabregat-Santiago, J. Bisquert, and T. W. Hamann, "Photoelectrochemical and Impedance Spectroscopic Investigation of Water Oxidation with Co-Pi-coated Hematite Electrodes," J. Am. Chem. Soc., 134 [40] 16693-700 (2012).

33. M. Tomkiewicz, "Impedance Spectroscopy of Rectifying Semiconductor/Electrolyte Interfaces," Electrochim. Acta, 35 [10] 1631-35 (1990).

34. J.-S. Lee and D.-Y. Kim, "Space-Charge Concepts on Grain Boundary Impedance of a High-Purity Yttria-Stabilized Tetragonal Zirconia Polycrystal," J. Mater. Res., 16 [9] 2739-51 (2001).

35. K. S. Cole and R. H. Cole, "Dispersion and Absorption in Dielectrics I. Alternating Current Characteristics," J. Chem. Phys., 9 [4] 341-51 (1941).

36. S. Havriliak and S. Negami, "A Complex Plane Analysis of $\alpha$-Dispersions in Some Polymer Systems," J. Polymer Sci., 14 [1] 99-117 (1966).

37. D. Losee, "Admittance Spectroscopy of Impurity Levels in Schottky Barriers,” J. Appl. Phys., 46 [5] 2204-14 (1975).

38. G. Crosbie, "Chemical Diffusivity and Electrical Conductivity in $\mathrm{TiO}_{2}$ containing a Submicron Dispersion of $\mathrm{SiO}_{2}$," J. Solid State Chem., 25 [4] 367-78 (1978).

39. J. Bisquert, "Influence of the Boundaries in the Impedance of Porous Film Electrodes," Phys. Chem. Chem. Phys., 2 [18] 4185-92 (2000).

40. E.-C. Shin, J. Ma, P.-A. Ahn, H.-H. Seo, D. T. Nguyen, and J. S. Lee, "Deconvolution of Four Transmission-LineModel Impedances in Ni-YSZ/YSZ/LSM Solid Oxide Cells 
and Mechanistic Insights," Electrochim. Acta, 188 240-53 (2016).

41. J. Mizusaki, "Model for Solid Electrolyte Gas Electrode Reaction Kinetics; Key Concepts, Basic Model Construction, Extension of Models, New Experimental Techniques for Model Confirmation, and Future Prospects," Electrochem., 82 [10] 819-29 (2014).

42. J. R. Macdonald, LEVM/LEVMW manual ver. 8.12 (June 2013).

43. J. Bisquert, G. Galcia-Belmonte, F. Fabregat-Santiago, N. S. Ferriols, P. Bogdanoff, and E. C. Pereira, "Doubling Exponent Models for the Analysis of Porous Film Electrodes by Impedance. Relaxation of $\mathrm{TiO}_{2}$ Nanoporous in Aqueous Solution," J. Phys. Chem. B, 104 [10] 2287-98 (2000).

44. J. R. Macdonald, "Impedance Spectroscopy," Ann. Biomed. Eng., 20 289-305 (1992).

45. J.-S. Lee, E.-C. Shin, D.-K. Shin, Y. Kim, P.-A. Ahn, H.-H. Seo, J.-M. Jo, J.-H. Kim, G.-R. Kim, Y.-H. Kim, J.-Y. Park, C.-H. Kim, J.-O. Hong, and K.-H. Hur, "Impedance Spectroscopy Models for X5R Multilayer Ceramic Capacitors," J. Korean Ceram. Soc., 49 [5] 475-83 (2012).

46. J. Moon, J.-A. Park, S.-J. Lee, J.-I. Lee, T. Zyung, E.-C. Shin, and J.-S. Lee, "A Physicochemical Mechanism of Chemical Gas Sensors Using an AC Analysis," Phys. Chem. Chem. Phys., 15 [23] 9361-74 (2013).

47. E.-C. Shin, P.-A. Ahn, H.-H. Seo, J.-M. Jo, S.-D. Kim, S.K. Woo, J.-H. Yu, J. Mizusaki, and J.-S. Lee, "Polarization Mechanism of High Temperature Electrolysis in a NiYSZ/YSZ/LSM Solid Oxide Cell by Parametric Impedance Analysis," Solid State Ionics, 232 80-96 (2013).
48. E.-C. Shin, P.-A. Ahn, H.-H. Seo, and J.-S. Lee, "Application of a General Gas Electrode Model to Ni-YSZ Symmetric Cells: Humidity and Current Collector Effects," J. Korean Ceram. Soc., 53 [5] 511-20 (2016).

49. J.-S. Lee, J. Jamnik, and J. Maier, "Generalized Equivalent Circuits for Mixed Conductors: Silver Sulfide as a Model System," Monat. Chem. (Chemical Monthly), 140 [9] 1113-19 (2009).

50. P.-A. Ahn, E.-C. Shin, G.-R. Kim, and J.-S. Lee, "Application of Generalized Transmission Line Models to Mixed Ionic-Electronic Transport Phenomena," J. Korean Ceram. Soc., 48 [6] 549-58 (2011).

51. P.-A. Ahn, E.-C. Shin, J.-M. Jo, J.-H. Yu, S.-K. Woo, and J.-S. Lee, "Mixed Conduction in Ceramic Hydrogen/Steam Electrodes by Hebb-Wagner Polarization in the Frequency Domain," Fuel Cells, 12 [6] 1070-84 (2012).

52. J. Maier, Physical Chemistry of Ionic Materials: Ions and Electrons in Solids; John Wiley \& Sons, 2004.

53. C. He, Z. Zheng, H. Tang, L. Zhao, and F. Lu, "Electrochemical Impedance Spectroscopy Characterization of Electron Transport and Recombination in $\mathrm{ZnO}$ Nanorod Dye-Sensitized Solar Cells,” J. Phys. Chem. C, 113 [24] 10322-25 (2009).

54. C. Fabrega, T. Andreu, A. Tarancón, C. Flox, A. Morata, L. CalvoBarrio, and J. Morante, "Optimization of Surface Charge Transfer Processes on Rutile $\mathrm{TiO}_{2}$ Nanorods Photoanodes for Water Splitting," Int. J. Hydrogen Energy, 38 [7] 2979-85 (2013).

55. H. Seo, K. H. Cho, H. Ha, S. Park, J. S. Hong, K. Jin, and K. T. Nam, "Water Oxidation Mechanism for 3d Transition Metal Oxide Catalysts under Neutral Condition," J. Korean Ceram. Soc., 54 [1] 1-8 (2017). 